\title{
Susceptible-Infectious-Recovered Models Revisited: From the Individual Level to the Population Level
}

\author{
Pierre Magal ${ }^{a}$ AND Shigui Ruan ${ }^{b, *}$ \\ ${ }^{a}$ Institut de Mathématiques de Bordeaux, UMR CNRS 5251 -Case 36, \\ Université Victor Segalen Bordeaux 2, \\ 3ter place de la Victoire, 33000 Bordeaux Cedex, France \\ ${ }^{b}$ Department of Mathematics, University of Miami, \\ Coral Gables, FL 33124-4250, USA
}

February 5, 2014

\begin{abstract}
The classical susceptible-infectious-recovered (SIR) model, originated from the seminal papers of Ross [51] and Ross and Hudson [52, 53] in 19161917 and the fundamental contributions of Kermack and McKendrick $[36,37,38]$ in 1927-1932, describes the transmission of infectious diseases between susceptible and infective individuals and provides the basic framework for almost all later epidemic models, including stochastic epidemic models using Monte Carlo simulations or Individual-Based Models (IBM). In this paper, by defining the rules of contacts between susceptible and infective individuals, the rules of transmission of diseases through these contacts, and the time of transmission during contacts, we provide detailed comparisons between the classical deterministic SIR model and the IBM stochastic simulations of the model. More specifically, for the purpose of numerical and stochastic simulations we distinguish two types of transmission processes: that initiated by susceptible individuals and that driven by infective individuals. Our analysis and simulations demonstrate that in both cases the IBM converges to the classical SIR model only in some particular situations. In general, the classical and individualbased SIR models are significantly different. Our study reveals that the timing of transmission in a contact at the individual level plays a crucial role in determining the transmission dynamics of an infectious disease at the population level.
\end{abstract}

Keywords: Susceptible-infectious-recovered (SIR) model, individual-based model (IBM), random graph of connection, numerical simulation, formal singular limit.

${ }^{*}$ Research was partially supported by the National Science Foundation grant DMS-1022728. 


\section{Introduction}

Mathematical modeling in epidemiology started with the pioneering work of Bernoulli [10] in 1760 in which he aimed at evaluating the effectiveness of inoculation against smallpox. The model of Bernoulli described the susceptible and recovered classes and already incorporated the chronological age of individuals (see Dietz and Heesterbeek [20, 21]). The susceptibleinfectious-recovered (SIR) model as we know today takes its origin in the fundamental works on "a priori pathometry" by Ross [51] and Ross and Hudson $[52,53]$ in 1916-1917 in which a system of ordinary differential equations was used to describe the transmission of infectious diseases between susceptible and infected individuals. In 1927-1933, Kermack and McKendrick [36, 37, 38] extended Ross's ideas and model, proposed the cross quadratic term $\beta I S$ linking the sizes of the susceptible (S) and infective (I) populations from a probabilistic analysis of the microscopic interactions between infective agents and/or vectors and hosts in the dynamics of contacts, and established the threshold theorem. Since then epidemic models have been extensively developed in several directions, we refer to the monographs of Bailey [7], Bartlett [9], Muench [45], Anderson and May [4], Busenberg and Cooke [13], Capasso [14], Murray [46], Daley and Gani [16], Mode and Sleeman [47], Brauer and Castillo-Chavez [11], Diekmann and Heesterbeek [19], Thieme [59], and Keeling and Rohani [35] on these topics.

In order to focus on the dynamical properties of an infectious disease itself, here we neglect the demography, namely the birth and death processes, and the immigration/emigration process. The classical SIR model takes the following form (Anderson and May [4]):

$$
\left\{\begin{array}{l}
S^{\prime}=-\beta \frac{S I}{N} \\
I^{\prime}=\beta \frac{S I}{N}-\eta_{R} I \\
R^{\prime}=\eta_{R} I,
\end{array}\right.
$$

where $S(t)$ is the number of susceptible individuals, $I(t)$ is the number of infective individuals (i.e. individuals who are infected and capable to transmit the disease), $R(t)$ is the number of recovered individuals at time $t$, respectively, and $N$ is the total number of individuals in the population. The parameter $\beta>0$ is called the infection rate (i.e. the contact rate times the probability of infection, see Thieme [59]), and $\eta_{R}>0$ is the recovery rate (i.e. the rate at which infective individuals recover). The SIR model has been used successfully to describe several epidemics (see for example [15]), but as far as we understand, this rate of infection is only derived empirically, namely by comparison of the model with real data.

When one neglects the demography, an epidemic model becomes a combination of the following aspects:

(a) a rule of contacts between individuals;

(b) a rule of transmission per contact;

(c) a rule of development of the infection at the level of individuals.

Since the development of an infection is not instantaneous, rule (c) can be described by introducing a latency between the transmission of 
the pathogen and the moment at which an exposed individual becomes capable to transmit the infection (namely becomes infective). This latency can be described by using either an extra exposed class (when the time of latency follows an exponential law), which leads to SEIR models, or an age of infection (i.e. the time since infection), which leads to age-structured models, we refer to Webb [61], Iannelli [33], Thieme [59], Magal et al. [41] for details on this topic. In this article, we will neglect the aspect (c) and focus only on (a) and (b).

In an epidemic of an infectious disease, the graph of contact plays a crucial role in the transmission of the disease. It is usually admitted (see Anderson and May [4] and Hethcote [30]) that the SIR model (1.1) is derived by using a "fully mixed" population. This means that all individuals have the same probability to contact with any other individuals in the population. Here we will see that even with a fully mixed population, the SIR model may fail to reproduce the dynamics of the epidemic. Actually we will see that more sophisticated models are needed to understand the dynamical property of an epidemic.

Of course in most epidemics, the contacts between individuals will arise only locally in space. Therefore more general graphs of contact are needed, we refer to Durrett and Levin [26], Newman [48], Durrett [24, 25], Meyers [43], Barrat et al. [8] (and references therein) for more information on this subject. Actually the space can be incorporated by using different approaches: it can be regarded as a continuous domain (see Rass and Radcliffe [50], Ruan [54], Ruan and $\mathrm{Wu}$ [55]) or again as a network (see Arino [6] and references therein). In this article, we will neglect the space in order to focus on the classical SIR model.

Stochastic individual-based models (IBM) have been extensively used to investigate threshold conditions and to evaluate the efficacy of disease control measures in which each host is viewed as an individual agent whose status changes based on probabilistic events occurring over time. IBM are particularly suitable to describe the transmission of infectious diseases in a small population in which the individual behavior plays an important role in the spread of diseases (DeAngelis and Mooij [18], Grimm and Railsback [29], Levin and Durrett [40], Keeling and Grenfell [34]). Studies have been performed to compare different types of IBM. For instance, Smieszek et al. [57] compared two different types of individual-based models, one assumes random mixing without repetition of contacts and the other assumes that the same contacts repeat day-by-day. They tested and compared how the total size of an outbreak differs between these model types depending on the key parameters such as transmission probability, number of contacts per day, duration of the infectious period, different levels of clustering and varying proportions of repetitive contacts. If the number of contacts per day is high or if the per-contact transmission probability is high, as seen in typical childhood diseases such as measles, they showed that random mixing models provide acceptable estimates of the total outbreak size. If the number of daily contacts or the transmission probability is low, such as the infection of meticillin-resistant Staphylococcus aureus (MRSA), they found that particular consideration should be given to the actual structure of potentially contagious contacts when designing the model. See also the comparison of a stochastic agent-based model and a structured metapop- 
ulation stochastic model for the progression of a baseline pandemic event in Italy by Ajelli et al. [1].

We should mention that the Gillespie algorithm or Doob-Gillespie algorithm (see Doob [22, 23] and Gillespie [27, 28]) provides a method to run random Monte-Carlo simulations associated to ordinary differential equations (see Andersson and Britton [5] and Kurtz [39]). This method was successfully used for chemical or biochemical systems of reactions. In epidemics, we will see in this article that changing the moment of pathogen's transmission from the beginning to the end of contact may influence the dynamical property of the equations.

The main issue to be addressed in this article is the comparison between the classical deterministic SIR model and its computer stochastic versions. The stochastic models will be derived by using Monte Carlo simulations or IBM. The increase in behavioral details provided by IBM, however, leads to much greater computational intensity and much greater difficulty in analyzing the significance of parameters. Some comparison between deterministic models and IBM have been performed by Pascual and Levin [49] (in the context of predator-prey), D'Agata et al. [17] (in the context of epidemics), Hinow et al. [32] (in the context of cell population dynamics), and Sharkey [56] (in the context of epidemics in networks). But as we will see, even with rather simple rules (a) and (b), the comparison between the SIR model (1.1) and the IBM derived from these stochastic rules (at the individual level) is not clear in general. Actually we will see that more general classes of SIR models are necessary to derive a comparison with the IBM.

The paper is organized as follows. In section 2 we make some assumptions about the rules of contacts between susceptible and infective individuals, the rules of transmission of diseases through these contacts, and the time of transmission during contacts. In section 3 we analyze the transmission driven only by susceptible individuals and compare the numerical simulations between the classical SIR model and the IBM. In section 4 , the transmission driven only by infective individuals is modeled and analyzed. Our analysis and simulations demonstrate that in both cases, the IBM converges to the classical SIR model only in some particular situations. In general, the classical SIR model and the IBM are significantly different. A brief discussion is given in section 5 .

\section{Rules of Contacts and Transmission}

In this section we present the stochastic process describing contacts between individuals. This process will lead to the construction of a simple deterministic model. The contacts are supposed to be arbitrarily given at an initial time, and in order to describe the evolution of the contacts with time, we will use the following rules.

We would like to point out that the evolution of the contact network is indeed dynamic since it changes with time. We define the rules of contacts, the rules of transmission, and the time of transmission for the purpose of numerical and stochastic simulations of the SIR model. These rules may not affect the outcome of an epidemic from a deterministic 
modeling point of view. However, they are important in numerical and stochastic simulations and produce dramatically different results.

\subsection{Rules of Contacts}

Firstly, we make some assumptions on the rules of contacts.

(a) At any time each individual has initiated exactly one contact with an individual in the population (possibly himself).

(b) The duration of a contact follows an exponential law and the average duration of a contact is $T_{C}>0$.

(c) At the end of a given contact the initiating individual randomly chooses a new individual within the population and the duration for this contact is determined.

The rules of contacts are chosen to be as simple as possible, since our goal is to obtain a comparison between an epidemic model using the above rules for contacts and the usual SI model. The duration of a given contact is the time from when the contact begins until the individual who initiated the contact concludes it and begins a new contact. Therefore the average contact duration $T_{C}>0$ can be estimated in practice. Indeed, $\nu_{C}=1 / T_{C}$ is the average number of contacts per unit of time.

In real epidemics, participants may have no choice on contact. Here the terminology "choose" or "initiate" means an individual of one type (either $S$ or $I$ type) is tracked through a time course of paired-contact with other individuals. In the following we will consider the extreme case where one follows either only the susceptible-individuals (abbr. $S$-individuals) or only the infectious-individuals (abbr. $I$-individuals). As we will see the model outcomes can be very different if the probability of transmission depends on the class of individuals that is tracked.

To further clarify the terminology, the above assumption also means that the graph of contact (in which the nodes or vertices are the individuals) is oriented. Moreover, we assume that each individual has one and only one outward arrow (possibly directed to himself) and at the end of a given oriented contact the new partner is chosen randomly (within the population). Oriented graphs have been used previously in the literature to describe epidemics (see for example Meyers et al. [44]). The definition of "choose" or "initiate" is contextual in the description of the graph (or the IBM).

In this model, an $S$-individual may have many contacts directed to him, in particular many directed contacts from an $I$-individuals and vice versa. The directed network approach may look at first more complicated, but it provides an advantage to construct an associated mathematical model to the epidemic considered (i.e. to evaluate the contacts between $S$ and $I$ individuals). One may also observe that similar treatment can be used for disease involving a criss-cross transmission between two populations (e.g. malaria, nosocomial infections, etc.). In such a situation, the probability of transmission may depend on which population is transmitting the pathogen. But the mathematical model associated with the problem will remain similar to the ones constructed here. Therefore, here 
it makes sense to look at the population divided into two subpopulations $S$ and $I$.

In order to describe the epidemics, we will classify the population into the class $S$ of susceptible individuals (i.e. capable to become infected by contact) and the class $I$ of infective individuals (i.e. capable to transmit the disease by contact). The transmission can only occur at the end of a contact between an $S$-individual and an $I$-individual. Moreover, we use the following rules of transmission. In order to take into account new infections, it will be appropriate to distinguish two processes:

(i) an $S$-individual chooses an I-individual for a contact;

(ii) an I-individual chooses an S-individual for a contact.

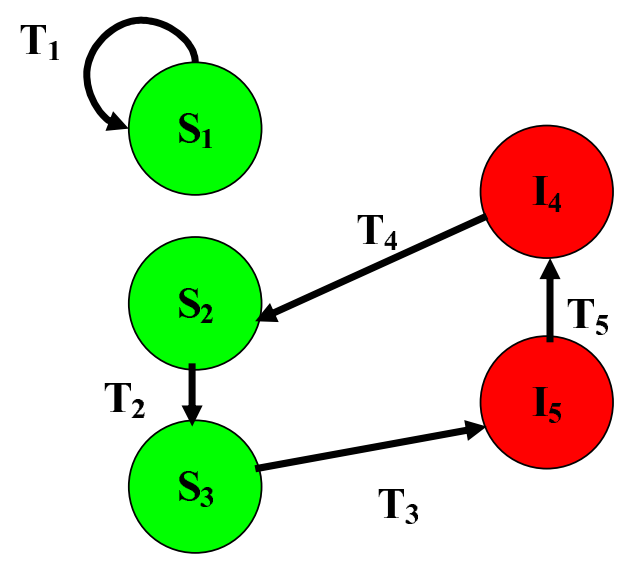

Figure 1: Diagram of the contact network at a given time $t \geq 0$. The first $S$-individual $\left(S_{1}\right)$ only contacts with himself. The second $S$-individual $\left(S_{2}\right)$ chooses to contact with a third $S$-individual $\left(S_{3}\right)$ who in turn contacts with an $I$-individual $\left(I_{5}\right) . I_{5}$ contacts another $I$-individual $\left(I_{4}\right)$ who in turn chooses $S_{2}$ for contact.

Depending on the disease, there may be an asymmetry between these processes and an important difference in their likelihood of leading to disease transmission. For example, if the infection is severe enough to immobilize infective individuals, then the transmission can only take place when susceptible individuals initiate contacts with infective individuals. On the other hand, for the common seasonal diseases, such as influenza, infected individuals continue to initiate contacts and they are very likely to play an important role in the transmission of the diseases.

As a first attempt to model this process, we first distinguish the number $S_{C}$ of $S$-individuals who choose a contact with an infective individual, the number $S_{F}$ of $S$-individuals who choose a contact with a susceptible individual, the number $I_{C}$ of $I$-individuals who choose a contact with a susceptible individual, and the number $I_{F}$ of $I$-individuals who choose a contact with an infective individual. A diagram of the contact network is given in Fig. 1. The arrows are pointing the individuals who are chosen for 
the contact. $T_{1}, T_{2}, \ldots, T_{5}$ are the times remaining to the end of the contact for each individual. For this graph of contacts, $\left(S_{c}, S_{f}, I_{c}, I_{f}\right)=(1,2,1,1)$. The individuals in $S_{c}$ and $I_{c}$ can be spotted by finding the arrows between susceptible and infective nodes.

Under the Rules of Contacts, we obtain the following model

$$
\left\{\begin{array}{l}
S_{C}^{\prime}=\nu_{C} \frac{I}{S+I}\left(S_{C}+S_{F}\right)-\nu_{C} S_{C} \\
S_{F}^{\prime}=\nu_{C} \frac{S}{S+I}\left(S_{C}+S_{F}\right)-\nu_{C} S_{F} \\
I_{C}^{\prime}=\nu_{C} \frac{S}{S+I}\left(I_{C}+I_{F}\right)-\nu_{C} I_{C} \\
I_{F}^{\prime}=\nu_{C} \frac{I}{S+I}\left(I_{C}+I_{F}\right)-\nu_{C} I_{F}
\end{array}\right.
$$

with

$$
S=S_{C}+S_{F} \text { and } I=I_{C}+I_{F} .
$$

In system $(2.1)$, the fraction $\frac{S(t)}{S(t)+I(t)}\left(\operatorname{resp} . \frac{I(t)}{S(t)+I(t)}\right)$ is the probability (in a "well mixed" population) that an individual initiates a new contact (resp. stops a contact) to a susceptible individual (resp. an infective) at a given time $t$. The quantity $\nu_{C} S_{C}(t)\left(\operatorname{resp} . \nu_{C} S_{F}, \nu_{C} I_{C}, \nu_{C} I_{F}\right)$ is the flux of individuals interrupting a contact at time $t$ in the class of $S_{C}$ (resp. $\left.S_{F}, I_{C}, I_{F}\right)$. Thus, in the absence of new infections, the rate that susceptible individuals forming new contacts is $\nu_{C}\left(S_{C}+S_{F}\right)$. So the rate at which susceptible individuals form new contacts with infective individuals is $\nu_{C} \frac{I}{S+I}\left(S_{C}+S_{F}\right)$, the flux into $S_{C}$. Similarly, the flux into $S_{F}$ is $\nu_{C} \frac{S}{S+I}\left(S_{C}+S_{F}\right)$. The equations for $I_{C}^{\prime}$ and $I_{F}^{\prime}$ can be explained similarly.

The following lemma is readily proved.

Lemma 2.1 For solutions of system (2.1), $S(t)$ and $I(t)$ are constant and

$\left(S_{C}(t), S_{F}(t), I_{C}(t), I_{F}(t)\right) \rightarrow\left(\frac{S I}{S+I}, \frac{S^{2}}{S+I}, \frac{S I}{S+I}, \frac{I^{2}}{S+I}\right)$ as $t \rightarrow+\infty$,

where the convergence is exponential.

The comparison between the ODE model (2.1) and Monte Carlo simulations of the model is given in Fig. 2. Here a Monte Carlo simulation means we run a stochastic computer program where we make a simulation of the above assumptions for $N$ individuals. In this figure we fix $\nu_{c}=1$, $I=N / 3, S=2 N / 3, I_{c}=0$ and $S_{c}=S$ at time $t=0$. Moreover, the number of individuals $N$ varied from $N=200$ in (a) up to $N=2000$ in (b). Therefore, when $N$ increases the solutions $S_{c}(t)$ and $I_{c}(t)$ of the stochastic simulations converge to the trajectories of the ordinary differential equation model (2.1). 
$(a)$

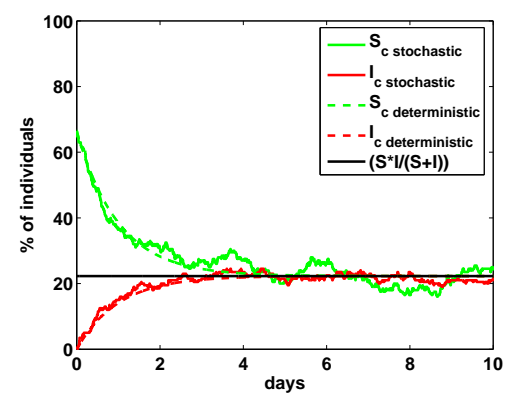

(b)

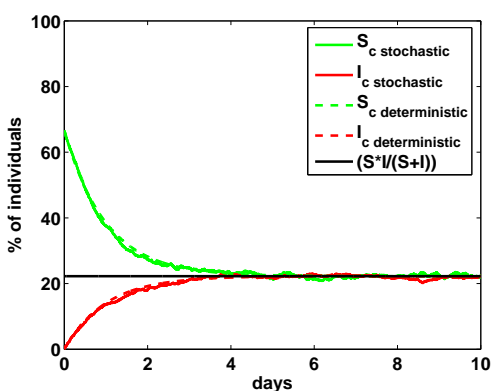

Figure 2: The comparison between solutions of the ordinary differential equation model (2.1) and Monte Carlo simulations of the model. The solutions of the stochastic model converge to the equilibrium solutions of the ODE model. Here $\nu_{c}=1, I=N / 3, S=2 N / 3, I_{c}=0$ and $S_{c}=S$ at time $t=0$. (a) $N=200$ and (b) $N=2000$.

We now specify the rules for disease transmission.

\subsection{Rules of Transmission}

We make assumptions about the rules of transmission: During a given contact between an S-individual and an I-individual, the probability of transmission is

(a) $p_{S} \in[0,1]$ if the contact was initiated by an $S$-individual;

(b) $p_{I} \in[0,1]$ if the contact was initiated by an I-individual.

We would like to make some remarks about $p_{S}$ and $p_{I}$. It is important to understand that the word "choosing" here serves only to construct the diagram of contact. For most cases (i.e. for non vector-borne diseases) there is no reason to assume that the transmission is oriented. Therefore, for non vector-borne diseases it will be natural to assume that $p_{S}=p_{I}$. Nevertheless in order to count the number of contacts between $S-$ and $I$-individuals it will be convenient to keep the word "choosing". For vector-borne or sexually transmitted diseases, $p_{S}$ and $p_{I}$ might be different. For example, it has been reported that (Higgins et al. [31]) the man-to-woman transmission rate of HIV/AIDS is different form the woman-to-man transmission rate. Also, in hospitals the contamination rate of health care workers and the colonization rate of patients for nosocomial infection are usually different. Thus, for the sake of generality we assume that $p_{S}$ and $p_{I}$ are different.

The next two sections are divided according to the following special cases:

(i) $p_{S}>0$ and $p_{I}=0$ (which will be called transmission driven by the susceptibles);

(ii) $p_{S}=0$ and $p_{I}>0$ (which will be called transmission driven by infectives). 
One may observe that case (i) actually corresponds to the fact that the contact initiated by $I$-individuals plays no role in the transmission. Therefore assumption (i) is also equivalent to supposing that only $S$ individuals are initiating contacts. Case (ii) is similar and this assumption is also equivalent to assuming that only $I$-individuals are initiating contacts. As we will see, even if these two cases look symmetric at first, these two scenarios are fairly different in terms of mathematical models. We also would like to note that $p_{S}$ and $p_{I}$ are used as the probabilities for all contacts during a simulation.

\subsection{Time of Transmission}

Finally we would like to address the issue on the time of transmission during a given contact. For a given contact between a susceptible individual and an infective individual, the transmission of the disease occurs (with a probability $p_{S}$ or $p_{I}$ ) only at one of the following two moments:

(c) the beginning of the contact;

(d) the end of the contact.

This is illustrated in Fig. 3. In sections 3.4 and 4.4 (on numerical simulations), we will examine the following two "extreme" cases. With the notations of Figure 3, case $(c)$ describes the situation where $t^{*}=t_{1}$ while case $(d)$ corresponds to the situation where $t^{*}=t_{2}$. Both cases may look very similar at first, in reality they are not. As we will see in sections 3.4 and 4.4 , case $(c)$ corresponds to the classical SIR model (1.1) while case $(d)$ describes new classes of SIR models which will be presented in sections 3 and 4 .

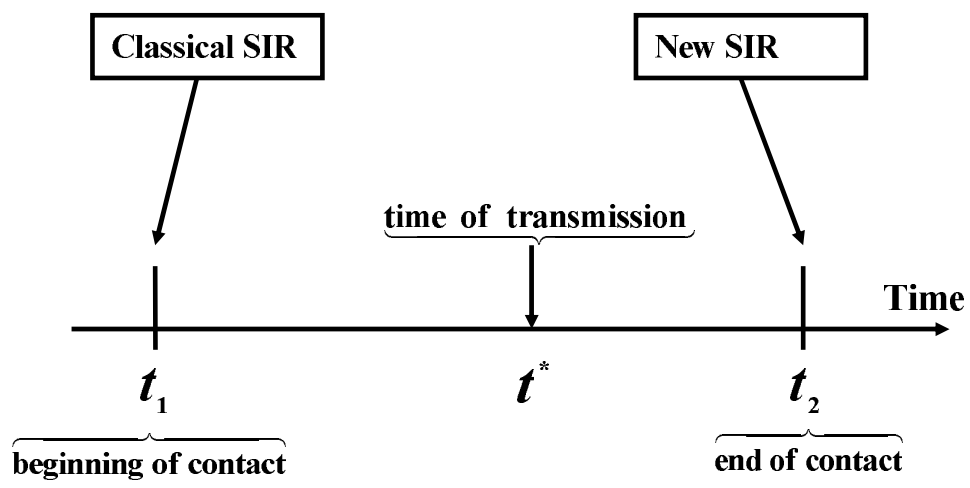

Figure 3: Time of transmission. (a) Transmission occurs at the beginning of a contact as assumed in the classical SIR model and (b) transmission occurs at the end of a contact as assumed in the new SIR model. In general, transmission can occur at any moment in between. 


\section{Transmission Driven only by $S$-individuals}

Under the Rules of contacts and Rules of transmission, we further assume that

$$
p_{S}>0 \text { and } p_{I}=0 .
$$

In addition we assume that the transmission occurs at the end of the contact period (i.e. Time of transmission (d) is satisfied). By combining these assumptions and using model (2.1), we obtain the following epidemic model

$$
\left\{\begin{array}{l}
S_{C}^{\prime}=\nu_{C}\left(\frac{I}{S_{F} I}\left(S_{C}+S_{F}-p_{S} S_{C}\right)-S_{C}\right) \\
S_{F}^{\prime}=\nu_{C}\left(\frac{S}{S+I}\left(S_{C}+S_{F}-p_{S} S_{C}\right)-S_{F}\right) \\
I^{\prime}=\nu_{C} p_{S} S_{C}
\end{array}\right.
$$

which yields

$$
\left\{\begin{array}{l}
S^{\prime}=-\nu_{C} p_{S} S_{C} \\
I^{\prime}=\nu_{C} p_{S} S_{C}
\end{array}\right.
$$

The terms in equations (3.1) involving $p_{S}$ come from the fact that the rates at which new contacts of susceptible individuals and infective individuals have changed due to the inclusion of disease transmission in (3.1). The flux of $S$-individuals ending a contact at time $t$ and in contact with an infective individual is $\nu_{C} S_{C}$. Therefore the flux of $S$-individuals becoming infected must be $p_{S} \nu_{C} S_{C}$. The rate at which susceptible ones are forming new contacts is now $\nu_{C}\left(S_{C}+S_{F}-p_{S} S_{C}\right)$.

\subsection{Model with recovery}

We consider the case that the population is divided into three groups: susceptible $S$, infective $I$, and recovered $R$. We also assume that only susceptible individuals initiate the contact.

Assumption 3.1 The duration of an infection follows an exponential law and the average duration of an infection is $T_{R}>0$.

Under the above assumption, the rate at which $I$-individuals are recovering is $\eta_{R}:=\frac{1}{T_{R}}$ and we obtain the following model

$$
\left\{\begin{array}{l}
S_{C}^{\prime}=\nu_{C}\left(\frac{I}{N}\left[S_{F}+\left(1-p_{S}\right) S_{C}\right]-S_{C}\right) \\
S_{F}^{\prime}=\nu_{C}\left(\frac{S+R}{N}\left[S_{F}+\left(1-p_{S}\right) S_{C}\right]-S_{F}\right) \\
I^{\prime}=\nu_{C} p_{S} S_{C}-\eta_{R} I \\
R^{\prime}=\eta_{R} I,
\end{array}\right.
$$

where $N=S+I+R$. The fluxes between the compartments of the model (3.2) are described in Fig. 4. 


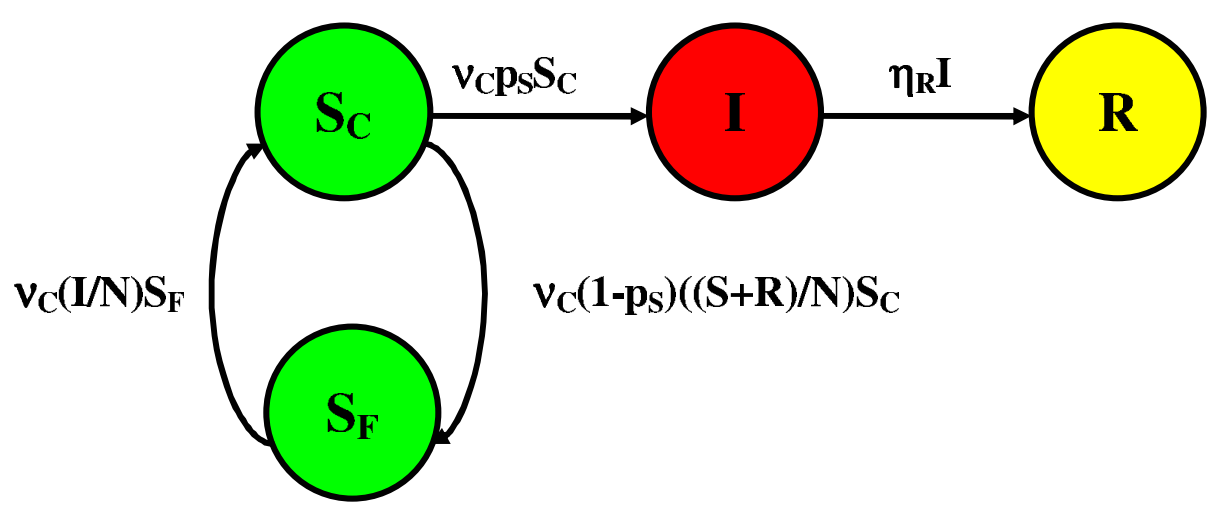

Figure 4: The flux diagram of model (3.2).

The parameters and state variables of the model are listed in Table 1.

Table 1. List of parameters and variables of the model

\begin{tabular}{|c|c|}
\hline Symbol & Interpretation \\
\hline$\nu_{C}$ & rate of contact \\
\hline$\eta_{R}$ & rate of recovery \\
\hline$T_{C}=1 / \nu_{C}$ & average duration of contacts \\
\hline$T_{R}=1 / \eta_{R}$ & average duration of infection \\
\hline$p_{S}$ & $\begin{array}{l}\text { probability of infection at the end of a contact } \\
\text { whenever a susceptible chooses an infective }\end{array}$ \\
\hline$p_{I}$ & $\begin{array}{l}\text { probability of infection at the end of a contact } \\
\text { whenever an infective chooses a susceptible }\end{array}$ \\
\hline$S_{C}$ & number of susceptibles in contact with an infective \\
\hline$S_{F}$ & number of susceptibles contact free with an infective \\
\hline$I_{C}$ & number of infectives in contact with a susceptible \\
\hline$I_{F}$ & number of infectives contact free with a susceptible \\
\hline$S:=S_{C}+S_{F}$ & number of susceptibles \\
\hline$I:=I_{C}+I_{F}$ & number of infectives \\
\hline$R$ & number of recovereds \\
\hline
\end{tabular}

\subsection{Asymptotic behavior}

Set $s=S / N, i=I / N, r=R / N, s_{c}=S_{c} / N$, and $s_{f}:=S_{f} / N$. Then model (3.2) is equivalent to

$$
\left\{\begin{array}{l}
s_{c}^{\prime}=\nu_{c}\left\{i\left(s-p_{S} s_{c}\right)-s_{c}\right\} \\
s_{f}^{\prime}=\nu_{c}\left\{(s+r)\left(s-p_{S} s_{c}\right)-s_{f}\right\} \\
i^{\prime}=\nu_{c} p_{S} s_{c}-\eta_{R} i \\
r^{\prime}=\eta_{R} i
\end{array}\right.
$$


By summing up the first two equations in system (3.3) we obtain the following model

$$
\left\{\begin{array}{l}
s^{\prime}=-\nu_{c} p_{S} s_{c} \\
i^{\prime}=\nu_{c} p_{S} s_{c}-\eta_{R} i \\
r^{\prime}=\eta_{R} i \\
s_{c}^{\prime}=\nu_{c}\left\{i\left(s-p_{S} s_{c}\right)-s_{c}\right\}
\end{array}\right.
$$

with $s(0) \geq 0, i(0) \geq 0, r(0) \geq 0$ and

$$
s(0)+i(0)+r(0)=1 \text { and } s_{c}(0) \in[0, s(0)] .
$$

Now consider $\bar{s}_{c}=\frac{s_{c}}{s}$ which is the probability (or the fraction) of $S$ individuals in contact with an $I$-individual within the population of $S$ individuals. By using the first equation of system (3.3) and system (3.4), we have

$$
\begin{aligned}
\bar{s}_{c}^{\prime} & =\frac{s_{c}^{\prime}}{s}-\frac{s_{c}}{s} \frac{s^{\prime}}{s}=\nu_{c} \frac{1}{s}\left\{i\left(s-p_{S} s_{c}\right)-s_{c}\right\}+\frac{s_{c}}{s} \frac{\nu_{c} p_{S} s_{c}}{s} \\
& =\nu_{c}\left\{i\left(1-p_{S} \bar{s}_{c}\right)-\bar{s}_{c}+p_{S} \bar{s}_{c}^{2}\right\} .
\end{aligned}
$$

Hence

$$
\bar{s}_{c}^{\prime}=\nu_{c}\left(i-\bar{s}_{c}\right)\left(1-p_{S} \bar{s}_{c}\right) .
$$

So we obtain the following system

$$
\left\{\begin{array}{l}
s^{\prime}=-\nu_{c} p_{S} \bar{s}_{c} s \\
i^{\prime}=\nu_{c} p_{S} \bar{s}_{c} s-\eta_{R} i \\
r^{\prime}=\eta_{R} i \\
\bar{s}_{c}^{\prime}=\nu_{c}\left(i-\bar{s}_{c}\right)\left(1-p_{S} \bar{s}_{c}\right),
\end{array}\right.
$$

where the initial values satisfy

$$
s(0)=s_{0} \geq 0, i(0)=i_{0} \geq 0, r(0)=r_{0} \geq 0 \text { and } \bar{s}_{c}(0)=\bar{s}_{c 0} \in[0,1)
$$

with $s_{0}+i_{0}+r_{0}=1$. We can also see that

$$
s(t)+i(t)+r(t)=1, \forall t \geq 0 .
$$

Moreover, we have the following inequality

$$
\bar{s}_{c}^{\prime}(t) \leq \nu_{c}\left(1-\bar{s}_{c}(t)\right)\left(1-p_{S} \bar{s}_{c}(t)\right)
$$

whenever $\bar{s}_{c}(t) \leq 1$. Hence we deduce that

$$
\bar{s}_{c}(t) \in(0,1), \forall t>0 .
$$

The last tool to complete the description of the asymptotic behavior is the following equality

$$
\frac{d}{d t}\left\{s+i-\frac{\eta_{R}}{p_{S} \nu_{c}}\left[\ln \left(1-p_{S} \bar{s}_{c}\right)+\ln (s)\right]\right\}=0 .
$$


Remark 3.2 The model does not coincide with the classical SIR model. Indeed, if $\eta_{R}=0$ then we have $s+i=1$ (for both models), it is clear that the equations

$$
\bar{s}_{c}^{\prime}=\nu_{c}\left(i-\bar{s}_{c}\right)\left(1-p_{S} \bar{s}_{c}\right)
$$

and

$$
i^{\prime}=\beta(1-i) i
$$

do not coincide. If $\eta_{R}>0$, equation (3.10) provides a formula for $\bar{s}_{c}$ which is not proportional to $i$.

As for the classical SIR model, equality (3.10) is the main tool to determine the limits of the $s$ and $r$ components in model (3.7). The equilibria of system (3.7) satisfy

$$
s^{*}+r^{*}=1 \text { and } i^{*}=\bar{s}_{c}^{*}=0
$$

By using the previous observations (3.8)-(3.10), we obtain the following proposition.

Proposition 3.3 Assume that $p_{S} \in(0,1), \nu_{c}>0, \eta_{R}>0$, and further suppose that

$$
s_{0}>0 \text { and } i_{0}>0 .
$$

Then all solutions of model (3.7) and their limits satisfy the following property for initial values with $i_{0}>0$

$$
\lim _{t \rightarrow+\infty}\left(\begin{array}{c}
s(t) \\
i(t) \\
r(t) \\
\bar{s}_{c}(t)
\end{array}\right)=\left(\begin{array}{c}
s^{*} \\
0 \\
r^{*} \\
0
\end{array}\right)
$$

with the equilibrium satisfying

$$
s^{*}+r^{*}=1
$$

and $s^{*}$ being the unique solution in $\left(0, \frac{\eta_{R}}{p_{S} \nu_{c}}\right]$ of the equation

$$
s^{*}-\frac{\eta_{R}}{p_{S} \nu_{c}} \ln \left(s^{*}\right)=\left\{s_{0}+i_{0}-\frac{\eta_{R}}{p_{S} \nu_{c}}\left[\ln \left(1-p_{S} \bar{s}_{c 0}\right)+\ln \left(s_{0}\right)\right]\right\} .
$$

Proof. Since $t \rightarrow s(t)$ is decreasing and $t \rightarrow r(t)$ is increasing, and both functions are bounded by 1 , we deduce that both functions converge (when $t$ goes to $+\infty)$, respectively, to $s^{*} \geq 0$ and $r^{*} \geq 0$. Moreover, since $s+i+r=1$ we deduce that $t \rightarrow i(t)$ also converges (when $t$ goes to $+\infty)$ to some $i^{*} \geq 0$. Now by using the $r$-equation one deduces that $i^{*}=0$ (otherwise $r(t)$ would be unbounded). Moreover, by using (3.10) one deduces that $s^{*}>0$ and satisfies (3.11). Now it remains to prove that $s^{*}$ belongs to $\left(0, \frac{\eta_{R}}{p_{S} \nu_{c}}\right]$. Assume that

$$
s^{*}>\frac{\eta_{R}}{p_{S} \nu_{c}} .
$$

Without loss of generality we assume that $i_{0}>0$ and $\bar{s}_{c 0}>0$. Otherwise if $\bar{s}_{c 0}=0$, since $i_{0}>0$ and

$$
\bar{s}_{c}^{\prime}=\nu_{c}\left(i-\bar{s}_{c}\right)\left(1-p_{S} \bar{s}_{c}\right),
$$


replacing $\left(i_{0}, \bar{s}_{c 0}\right)$ by any $\left(i(t), \bar{s}_{c}(t)\right)$ for $t>0$ we obtain the desired property.

Next by using the $i$-equation in (3.7) we obtain

$$
\left\{\begin{array}{l}
i^{\prime} \geq \eta_{R}\left(\bar{s}_{c}-i\right) \\
\bar{s}_{c}^{\prime}=\nu_{c}\left(i-\bar{s}_{c}\right)\left(1-p_{S} \bar{s}_{c}\right) .
\end{array}\right.
$$

We deduce that

$$
\left(i(t), \bar{s}_{c}(t)\right) \geq(x(t), y(t)), \forall t \geq 0,
$$

where $(x, y)$ is the solution of the monotone system (see Smith [58])

$$
\left\{\begin{array}{l}
x^{\prime}=\eta_{R}(y-x) \\
y^{\prime}=\nu_{c}(x-y)\left(1-p_{S} y\right)
\end{array}\right.
$$

with

$$
x(0)=i_{0}>0 \text { and } y(0)=\bar{s}_{c 0}>0 .
$$

Set

$$
\varepsilon=\min \left(i_{0}, \bar{s}_{c 0}\right)
$$

We conclude that

$$
\lim _{t \rightarrow+\infty} i(t) \geq \varepsilon \text { and } \lim _{t \rightarrow+\infty} \bar{s}_{c}(t) \geq \varepsilon,
$$

since $(\varepsilon, \varepsilon)$ is an equilibrium of system (3.13), we obtain a contradiction with the fact that

$$
\lim _{t \rightarrow+\infty} i(t)=\lim _{t \rightarrow+\infty} \bar{s}_{c}(t)=0
$$

This completes the proof.

\subsection{Comparison with the classical SIR model}

Let $\beta_{S}>0$ and $\eta_{R}>0$ be fixed. Set $\nu_{c}=\frac{1}{\varepsilon}, p_{S}=\beta_{S} \varepsilon$, where $\varepsilon \in\left(0, \beta_{S}^{-1}\right]$ is a small parameter. By using this rescaling, system (3.7) becomes the following system parameterized by $\varepsilon$ :

$$
\left\{\begin{array}{l}
s_{\varepsilon}^{\prime}=-\beta_{S} \bar{s}_{c \varepsilon} s_{\varepsilon} \\
i_{\varepsilon}^{\prime}=\beta_{S} \bar{s}_{c \varepsilon} s_{\varepsilon}-\eta_{R} i_{\varepsilon} \\
r_{\varepsilon}^{\prime}=\eta_{R} i_{\varepsilon} \\
\bar{s}_{c \varepsilon}^{\prime}=\frac{1}{\varepsilon}\left(i_{\varepsilon}-\bar{s}_{c \varepsilon}\right)\left(1-\beta_{S} \varepsilon \bar{s}_{c \varepsilon}\right)
\end{array}\right.
$$

with

$$
s_{\varepsilon}(0)=s_{0}>0, i_{\varepsilon}(0)=i_{0}>0, r(0)=r_{0} \geq 0 \text { and } \bar{s}_{c c}(0)=\bar{s}_{c 0} \in[0,1) .
$$

Indeed when $\varepsilon$ goes to 0 we obtain a singular perturbation problem.

The main question to be addressed in this section is the convergence of the first three components of the system.

Now consider the classical SIR model

$$
\left\{\begin{array}{l}
s^{\prime}=\beta_{S} i s \\
i^{\prime}=\beta_{S} i s-\eta_{R} i \\
r^{\prime}=\eta_{R} i
\end{array}\right.
$$


with

$$
s(0)=s_{0}>0, i(0)=i_{0}>0, r(0)=r_{0} \geq 0 .
$$

Recall now the classical result of the SIR model (see Hethcote [30]). By using the fact that

$$
\frac{d}{d t}\left[s+i-\frac{\eta_{R}}{\beta_{S}} \ln (s)\right]=0
$$

and the same argument as above one has the following result.

Proposition 3.4 Assume that

$$
s_{0}>0 \text { and } i_{0}>0,
$$

then

$$
\lim _{t \rightarrow+\infty}\left(\begin{array}{c}
s(t) \\
i(t) \\
r(t)
\end{array}\right)=\left(\begin{array}{c}
s^{*} \\
0 \\
r^{*}
\end{array}\right),
$$

where $s^{*}$ is the unique solution in $\left(0, \frac{\eta_{R}}{\beta_{S}}\right]$ of the equation

$$
\left.s^{*}-\frac{\eta_{R}}{\beta_{S}} \ln \left(s^{*}\right)=\left\{s_{0}+i_{0}-\frac{\eta_{R}}{\beta_{S}} \ln \left(s_{0}\right)\right]\right\} .
$$

The main result of this section is the following theorem on the convergence of the solution.

Theorem 3.5 (Uniform convergence in time) Under the above assumptions, if

$$
s_{\varepsilon}(0)=s(0)>0, i_{\varepsilon}(0)=i(0)>0, r_{\varepsilon}(t)=r(0) \geq 0,
$$

then

$$
\left(\begin{array}{c}
s_{\varepsilon}(t) \\
i_{\varepsilon}(t) \\
r_{\varepsilon}(t)
\end{array}\right) \rightarrow\left(\begin{array}{c}
s(t) \\
i(t) \\
r(t)
\end{array}\right)
$$

as $\varepsilon \rightarrow 0$ uniformly with respect to $t$ in $[0,+\infty)$.

In other word, for each constant $\delta>0$, we can find $\widehat{\varepsilon}=\widehat{\varepsilon}(\delta)>0$, such that

$$
\left|s_{\varepsilon}(t)-s(t)\right| \leq \delta, \quad\left|i_{\varepsilon}(t)-i(t)\right| \leq \delta \text { and }\left|r_{\varepsilon}(t)-r(t)\right| \leq \delta
$$

for each $\varepsilon \in(0, \widehat{\varepsilon}]$ and each $t \geq 0$.

In order to prove this result, we start with a convergence result for a finite time.

Lemma 3.6 Let $\tau>0$. Then

$$
\left(\begin{array}{c}
s_{\varepsilon}(t) \\
i_{\varepsilon}(t) \\
r_{\varepsilon}(t)
\end{array}\right) \rightarrow\left(\begin{array}{c}
s(t) \\
i(t) \\
r(t)
\end{array}\right)
$$

as $\varepsilon \rightarrow 0$ uniformly with respect to $t$ in $[0, \tau]$. 
Proof. Let $\tau>0$ be fixed. It is clear the

$$
i_{\varepsilon}(t)+s_{\varepsilon}(t) \leq 1, \forall t \in[0, \tau],
$$

and by construction we have

$$
0 \leq s_{\varepsilon c}(t) \leq 1 \text { and } 0 \leq i_{\varepsilon c}(t) \leq 1 .
$$

We also have

$$
\begin{aligned}
s_{\varepsilon}^{\prime} & =-\beta_{S} \bar{s}_{c \varepsilon} s_{\varepsilon} \\
i_{\varepsilon}^{\prime} & =\beta_{S} \bar{s}_{c \varepsilon} s_{\varepsilon}-\eta_{R} i_{\varepsilon} \\
r_{\varepsilon}^{\prime} & =\eta_{R} i_{\varepsilon} .
\end{aligned}
$$

Thus

$$
\left|i_{\varepsilon}^{\prime}(t)\right|+\left|s_{\varepsilon}^{\prime}(t)\right|+\left|r_{\varepsilon}^{\prime}(t)\right| \leq 2\left(\beta_{S}+\eta_{R}\right), \forall t \in[0, \tau] .
$$

Therefore, by the Arzela-Ascoli theorem, for each sequence $\varepsilon_{n} \rightarrow 0$ we find a subsequence (denoted with the same index) such that

$$
s_{\varepsilon_{n}}(t) \rightarrow s(t), \quad i_{\varepsilon_{n}}(t) \rightarrow i(t) \text { and } r_{\varepsilon_{n}}(t) \rightarrow r(t) \text { as } n \rightarrow+\infty,
$$

uniformly with respect to $t$ in $[0, \tau]$. Moreover,

$$
\begin{aligned}
\bar{s}_{c \varepsilon}^{\prime} & =\frac{1}{\varepsilon}\left(i_{\varepsilon}-\bar{s}_{c \varepsilon}\right)\left(1-\beta_{S} \varepsilon \bar{s}_{c \varepsilon}\right) . \\
& =\frac{1}{\varepsilon}\left[i_{\varepsilon}\left(1-\beta_{S} \varepsilon \bar{s}_{c \varepsilon}\right)-\bar{s}_{c \varepsilon}\left(1-\beta_{S} \varepsilon \bar{s}_{c \varepsilon}\right)\right],
\end{aligned}
$$

so

$$
\bar{s}_{c \varepsilon}^{\prime}=-\frac{1}{\varepsilon} \bar{s}_{c \varepsilon}+\frac{1}{\varepsilon}\left[i_{\varepsilon}-\beta_{S} \varepsilon\left(\bar{s}_{c \varepsilon}-\bar{s}_{c \varepsilon}^{2}\right)\right]
$$

Therefore,

$\bar{s}_{\varepsilon c}(t)=e^{-\frac{t}{\varepsilon}} \bar{s}_{c 0}+\int_{0}^{t} \frac{1}{\varepsilon} e^{-\frac{(t-l)}{\varepsilon}} i_{\varepsilon}(l) d l-\int_{0}^{t} e^{-\frac{(t-l)}{\varepsilon}} \beta_{S}\left[\bar{s}_{c \varepsilon}(l)-\bar{s}_{c \varepsilon}^{2}(l)\right] d l$.

Since

$$
\int_{0}^{t} \frac{1}{\varepsilon_{n}} e^{-\frac{1}{\varepsilon_{n}}(t-l)} i_{\varepsilon_{n}}(l) d l \rightarrow i(t) \text { as } n \rightarrow+\infty \text { in } L^{1}(0, \tau)
$$

and

$e^{-\frac{t}{\varepsilon_{n}}} \bar{s}_{c 0}-\int_{0}^{t} e^{-\frac{1}{\varepsilon_{n}}(t-l)} \beta_{S}\left[\bar{s}_{c \varepsilon}(l)-\bar{s}_{c \varepsilon}^{2}(l)\right] d l \rightarrow 0$ as $n \rightarrow 0$ in $L^{1}(0, \tau)$,

the result follows.

Proof of Theorem 3.5. The proof is to combine the (monotone) convergence of $t \rightarrow s(t)$ and $t \rightarrow r(t)$, Lemma 3.6, and the convergence of the equilibrium as $\varepsilon \rightarrow 0$ (i.e. formula (3.11) and formula (3.17)). 


\subsection{Numerical simulations}

In order to compare these models numerically we consider some extreme cases. The codes used for the numerical simulations can be downloaded at http://www.math.u-bordeaux1.fr/ pmagal/SIR/SIR.htm.

Actually the IBM are rather delicate to run since some parameters and terms need to be more specific. Here we specify the rules of transmission between an $S$-individual and an $I$-individual.

Assumption 3.7 We further assume that

(a) (New SIR Model) For a given pair of $(S, I)$-individuals in contact, if the individual initiating the contact is an $S$-individual, then the transmission only occurs at the end of the contact with the probability $p_{S}$.

(b) (Classical SIR Model) For a given pair of $(S, I)$-individuals in contact, if the individual initiating the contact is an $S$-individual, then the transmission only occurs at the beginning of the contact with the probability $p_{S}$.

We will use Assumption 3.7 (a) to run an IBM (called IBM $_{11}$ here) which corresponds to model (3.2), while Assumption 3.7 (b) will be used to run an IBM (called $\mathrm{IBM}_{21}$ here) which corresponds to the classical SIR model (1.1). There are four cases in total which are summarized in Table 2. The two other cases will be studied in section 4.4. As we will see, replacing one model by the other might lead to a large bias in the prediction. Therefore one must be very careful in using IBM to simulate an epidemic.

Table 2. List of four IBMs.

\begin{tabular}{|c|c|c|}
\hline & Transmission driven by S & Transmission driven by I \\
\hline End of contact & $\mathrm{IBM}_{11}$ (First new SIR) & $\mathrm{IBM}_{12}$ (Second new SIR) \\
\hline Beginning of contact & $\mathrm{IBM}_{21}$ (Classical SIR) & $\mathrm{IBM}_{22}$ (Classical SIR) \\
\hline
\end{tabular}

\subsubsection{Simulations with a fully random graph of connection at time $t=0$}

In Fig. 5 we compare the new SIR model (3.2) and the classical SIR model (1.1) with $\beta=\beta_{S}=p_{S} / T_{C}$ fixed. The initial value $S_{C}=S I / N$ at time $t=0$, therefore the contacts are assumed to be already stabilized. We observe large bias in comparing both models. The deviation is confirmed in the numerical simulations of the IBM in Fig. 5. As predicted by Theorem 3.5, we first observe numerically the convergence of solutions of SIR model (3.2) to the solutions of the classical SIR model (1.1) when the average time of contact $T_{C}$ goes to zero. Note that the curve of $R(t)$, the number of recovered individuals, is influenced by the parameter $T_{C}$. Let $R_{\infty}$ be the limit of $R(t)$ when $t$ goes to $+\infty$. Recall that $R_{\infty}$ is the total number of cases produced by an epidemic. We can see that $R_{\infty}$ is influenced by the parameter $T_{C}$. In Fig. $5(\mathrm{a})$ the total numbers of cases of the new SIR model (3.2) and the classical SIR model (1.1) are 
fairly different. Here $S=0.9, I=0.1, S_{c}=S I /(S+I+R)$ at time $t=0 . T_{R}=5$ and $\beta_{S}=0.1$ were fixed. In (a) (resp. (b)), $T_{C}=10$ and $p_{S}=\beta_{S} T_{C}=1$ (resp. $T_{C}=1$ and $\left.p_{S}=\beta_{S} T_{C}=0.1\right)$ were fixed.

(a)

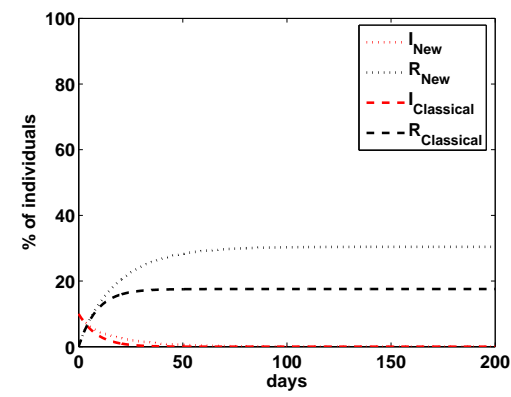

(b)

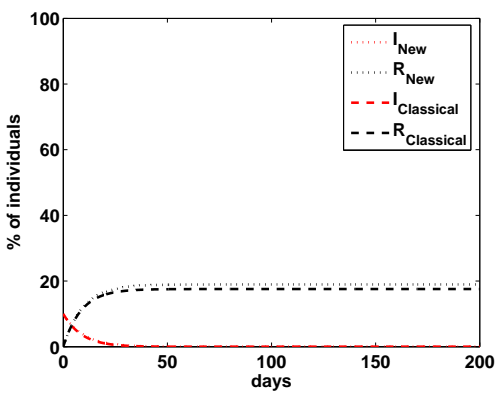

Figure 5: Comparison of the new SIR model (3.2) (dotted curves) and classical SIR model (1.1) (dashed curves) with $\beta=\beta_{S}=p_{S} / T_{C}, S=0.9, I=0.1, S_{c}=$ $S I /(S+I+R)$ at time $t=0 . T_{R}=5$ and $\beta_{S}=0.1$ were fixed. (a) $T_{C}=10$ and $p_{S}=\beta_{S} T_{C}=1$. (b) $T_{C}=1$ and $p_{S}=\beta_{S} T_{C}=0.1$. In both cases the solutions of the new SIR model converge to the solutions of the classical SIR model.

Comparison of the IBM, the new SIR model (3.2), and the classical SIR model (1.1) is presented in Fig. 6. Here $I=(1 / 10) N, S=(9 / 10) N$, with $S_{c}=S I /(S+I+R)$ at time $t=0 . T_{C}=5, T_{R}=5, \beta_{S}=0.2$ and $p_{S}=\beta_{S} T_{C}=1$ were fixed. $\nu_{c}=1 / T_{C}=1 / 5, \nu_{R}=1 / T_{R}=1 / 5$. $N$ (the total number of individuals in the IBM) varies from $N=100$ in (a) and (c) to $N=10000$ in (b) and (d). In (a) (resp. (b)) the $\mathrm{IBM}_{11}$ was run to simulate the transmission at the end of the contact for $N=100$ (resp. $N=10000$ ). In (c) (resp. (d)) the $I_{B M} M_{21}$ was run to simulate the transmission at the beginning of the contact for $N=100$ (resp. $N=10000)$. 
(a)

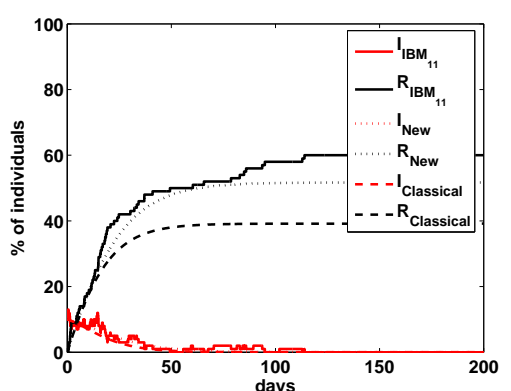

(c)

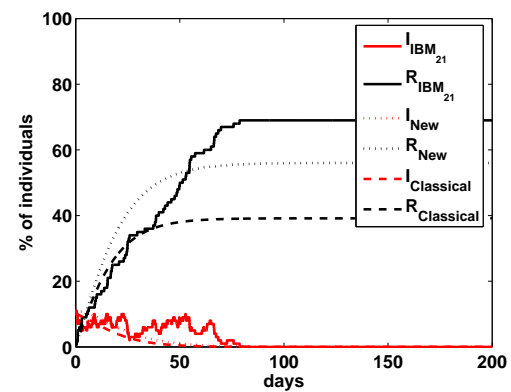

(b)

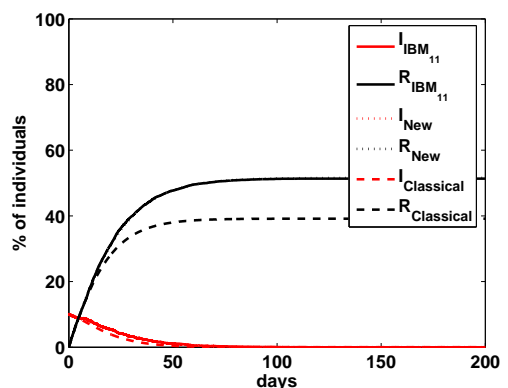

(d)

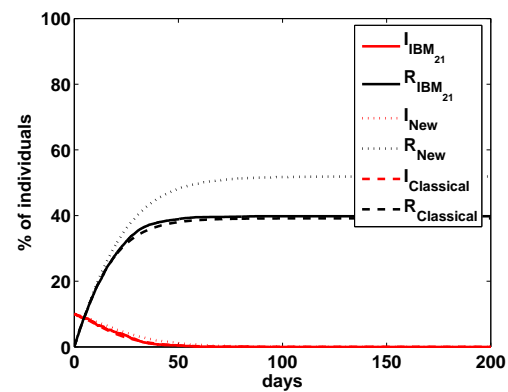

Figure 6: Comparison of simulations of the IBM (solid curves), the new SIR model (3.2) (dotted curves), and the classical SIR model (1.1) (dashed curves) with $\beta=\beta_{S}, I=(1 / 10) N, S=(9 / 10) N$ with $S_{c}=S I /(S+I+R)$ at time $t=0 . T_{C}=5, T_{R}=5, \beta_{S}=0.2$ and $p_{S}=\beta_{S} T_{C}=1$ were fixed. $\nu_{c}=1 / T_{C}=$ $1 / 5, \nu_{R}=1 / T_{R}=1 / 5$. In (a) and (b) the $\mathrm{IBM}_{11}$ was run to simulate the transmission at the end of the contact for $N=100$ and $N=10000$, respectively. In (c) and (d) the $\mathrm{IBM}_{21}$ was run to simulate the transmission at the beginning of the contact for $N=100$ and $N=10000$, respectively.

In order to illustrate the random fluctuations occurring in $\mathrm{IBM}_{11}$ and $\mathrm{IBM}_{21}$, we provide more numerical simulations of these two models with 50 runs. For the sake of simplicity, we only plot the simulations of the recovered class in see Fig. 7. In Fig. 7(a) (resp. (b)) the $\mathrm{IBM}_{11}$ was run 50 times to simulate the transmission at the end of the contact for $N=100$ (resp. $N=10000$ ). In Fig. 7 (c) (resp. (d)) the $\mathrm{IBM}_{21}$ was run 50 times to simulate the transmission at the beginning of the contact for $N=100$ (resp. $N=10000)$. All parameter values are the same as in Fig. 6 . 
(a)

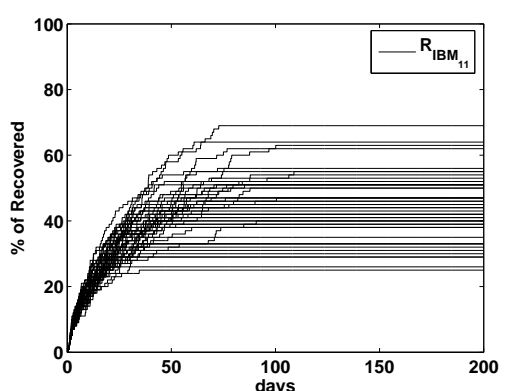

(c)

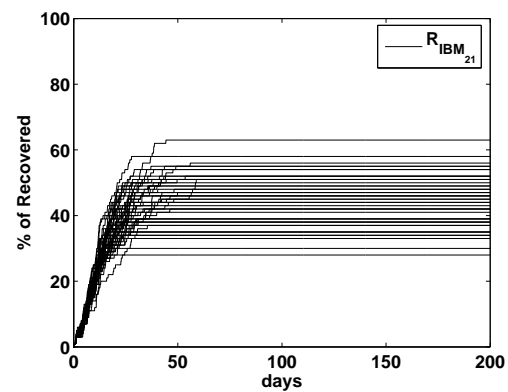

(b)

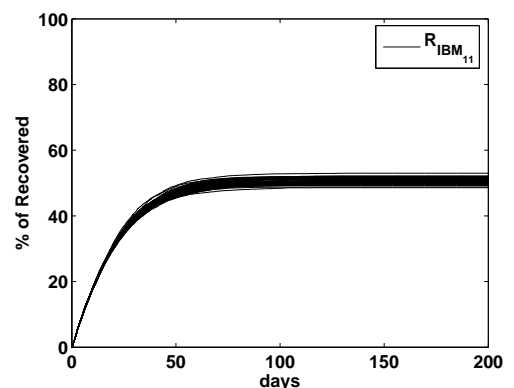

(d)

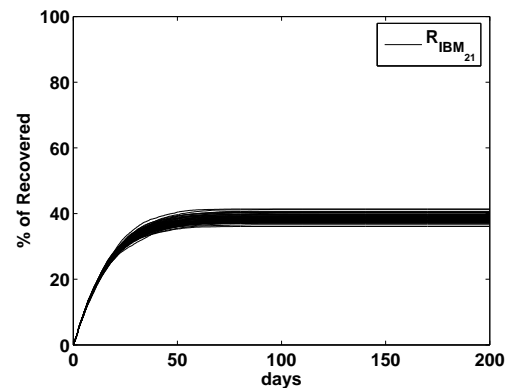

Figure 7: Simulations of the recovered class in the IMB. In (a) and (b) the $\mathrm{IBM}_{11}$ was run 50 times to simulate the transmission at the end of the contact for $N=100$ and $N=10000$, respectively. In (c) and (d) the $\mathrm{IBM}_{21}$ was run 50 times to simulate the transmission at the beginning of the contact for $N=100$ and $N=10000$, respectively. All parameters are the same as in Fig. 6 .

\subsubsection{Simulations with a non-fully random graph of con- nection at time $t=0$}

In this subsection we run some simulations assuming that $S_{C}=S$ at time $t=0$. This means that all $S$-individuals choose randomly a contact with an $I$-individual at $t=0$, and for $t>0$ all individuals choose randomly a contact within the all population (i.e. including the $S, I$ and $R$ individuals). This also means that the contacts are not yet stabilized at time $t=0$.

In Fig. 8 we observe a large deviation between the new SIR model (3.2) and the classical SIR model (1.1), but the former still predicts the $\mathrm{IBM}_{11}$ when the number of individuals increases; that is, the solutions of the $\mathrm{IBM}_{11}$ converge to the equilibrium solutions of the new SIR model (3.2) when the number of individuals increases. In both (a) and (b) the classical SIR model (1.1) (with $\beta=\beta_{S}:=p_{S} / T_{C}$ ) fails to predict the total number of cases produced by $\mathrm{IBM}_{11}$ (even when $T_{C}=0.1$ ). The classical SIR model (1.1) cannot predict this case, because the contacts are not yet stabilized, and it takes count of the evolution of contacts between individuals. Here $S=0.9, I=0.1, S_{c}=S$ at time $t=0 . T_{R}=5$ and 
$\beta_{S}=0.1$ are fixed. In (a) (resp. (b)) $T_{C}=10$ and $p_{S}=\beta_{S} T_{C}=1$ (resp. $T_{C}=0.1$ and $p_{S}=\beta_{S} T_{C}=0.1$ ) are fixed. In (a) and (b), the total number of individuals is fixed at $N=100$ and $N=1000$ respectively for the $\mathrm{IBM}_{11}$. Of course by taking $T_{C}$ smaller enough and $p_{S}=\beta_{S} T_{C}$, the total number of cases will finally be predicted by the classical SIR model. This question has to be explored further in order to derive some practical evaluation of the time of contacts and the probability of transmission in order to use the classical SIR model.

(a)

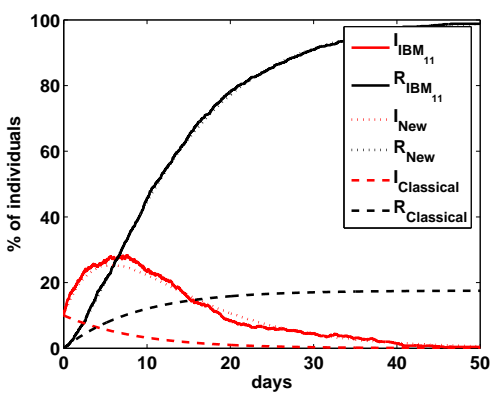

(b)

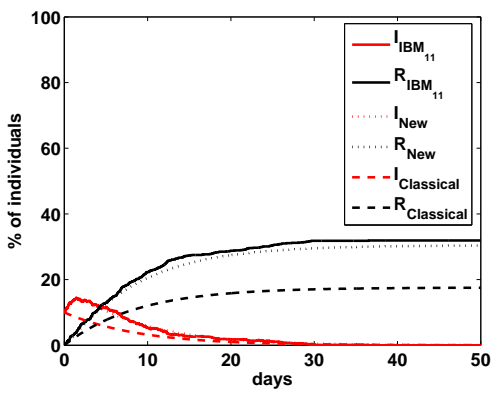

Figure 8: Comparison of simulations of the $\mathrm{IBM}_{11}$ (solid curves), the new SIR model (3.2) (dotted curves), and the classical SIR model (1.1) (dashed curves) with $\beta=\beta_{S}$ and a non-fully random graph of connection at time $t=0$. Here $S=0.9, I=0.1, S_{c}=S$ at time $t=0 . T_{R}=5$ and $\beta_{S}=0.1$ are fixed. (a) $T_{C}=10$ and $p_{S}=\beta_{S} T_{C}=1$. (b) $T_{C}=0.1$ and $p_{S}=\beta_{S} T_{C}=0.1$.

\section{Transmission Driven only by $I$-individuals}

\subsection{Gain and loss of contacts}

Under the Rules of contacts and Rules of transmission, we assume in addition that

$$
p_{S}=0 \text { and } p_{I}>0 .
$$

This is also equivalent to assuming that only the $I$-individuals are building some contacts with other individuals chosen randomly in the population.

We define $S_{n}(t)$ as the number of $S$-individuals that have been chosen $n$ times for a contact by $I$-individuals. So each $S$-individual in class $S_{n}$ has been chosen exactly $n$ times for a contact by exactly $n$ different infective individuals. The total number of $S$-individuals is given by

$$
S:=\sum_{n=0}^{+\infty} S_{n} .
$$

The structured diagram of the population in term of contacts is given in Fig. 9. Recall that each $I$-individual chooses at most one $S$-individual. Since $I$-individuals are choosing randomly an individual in the population at the end of each contact, an $S$-individual can be chosen from 0 to a 
number up to the number of $I$-individuals. The arrows are pointing to the group of $S$-individuals which have been chosen for a contact by $I$ individuals. $S_{0}$ is the group of $S$-individuals which has not been chosen by $I$-individuals, and $S_{n}$ is the group of $S$-individuals which has been chosen $n$ times by $I$-individuals, where $n=1,2, \ldots$

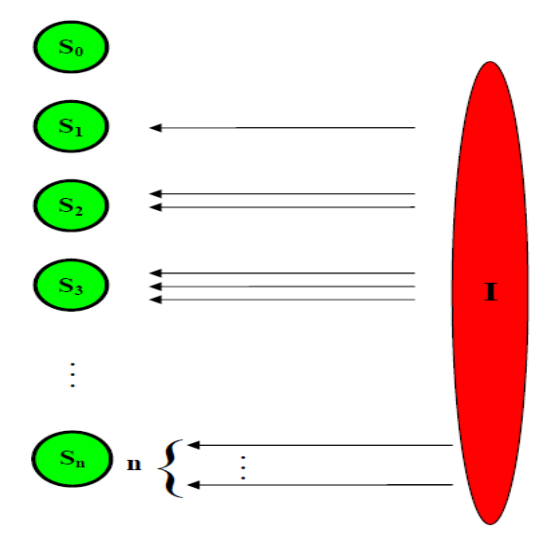

Figure 9: Structured diagram of the population in term of contacts indicating the number of $S$-individuals chosen for a contact by $I$-individuals. $S_{n}$ is the group of $S$-individuals which has been chosen $n$ times by $I$-individuals, where $n=0,1,2, \ldots$

In order to present the model, we first consider separately the following two processes for $S$-individuals: 1$)$ the gain contacts with $I$-individuals; 2 ) the loss contacts with $I$-individuals. To describe the processes, we first make the following assumption.

Gain of Contacts: Assume that, at any given time $t \geq 0$, each $S$ individual can gain at most one contact with an I-individual.

Under this assumption the model describing the gains of contacts is given by the following infinite system of ordinary differential equations:

$$
\left\{\begin{array}{l}
S_{0}^{\prime}=\nu_{c}\left[-\frac{I(t)}{N} S_{0}(t)\right] \\
S_{1}^{\prime}=\nu_{c}\left[\frac{I(t)}{N} S_{0}(t)-\frac{I(t)}{N} S_{1}(t)\right] \\
S_{2}^{\prime}=\nu_{c}\left[\frac{I(t)}{N} S_{1}(t)-\frac{I(t)}{N} S_{2}(t)\right] \\
\cdots \\
S_{n}^{\prime}=\nu_{c}\left[\frac{I(t)}{N} S_{n-1}(t)-\frac{I(t)}{N} S_{n}(t)\right] \\
\ldots
\end{array}\right.
$$

For $S$-individuals, the total number of contacts with $I$-individuals is given 
by $\sum_{n=0}^{+\infty} n S_{n}(t)$ and its variation is

$$
\begin{aligned}
\frac{d}{d t} \sum_{n=0}^{+\infty} n S_{n}(t) & =\nu_{c} \frac{I(t)}{N}\left[\sum_{n=1}^{+\infty} n\left(S_{n-1}(t)-S_{n}(t)\right)\right] \\
& =\nu_{c} \frac{I(t)}{N}\left[\sum_{n=0}^{+\infty}(n+1) S_{n}(t)-\sum_{n=1}^{+\infty} n S_{n}(t)\right] .
\end{aligned}
$$

Hence

$$
\frac{d}{d t} \sum_{n=0}^{+\infty} n S_{n}(t)=\nu_{c} \frac{I(t)}{N} S(t) .
$$

Now we shall remember that the variation of the number of contacts with $I$-individuals is $\nu_{c} I$. Since by assumption $I$-individuals are choosing their new contacts randomly, we shall consider the probability of finding an $S$-individual within the population (namely $\frac{S}{N}$ ). Therefore the rate at which $S$-individuals gain contacts with $I$-individuals must be $\nu_{c} I \frac{S}{N}$. This fully justifies model (4.2).

We also make the following additional assumption.

Loss of Contacts: Assume that, at any given time $t \geq 0$, each $S$ individual can lose at most one contact with an I-individual.

Then the model describing the loss of contacts of $S$-individuals with $I$ individuals is given by the following infinite system of ordinary differential equations:

$$
\left\{\begin{array}{l}
S_{0}^{\prime}=\nu_{c}\left[S_{1}(t)\right] \\
S_{1}^{\prime}=\nu_{c}\left[2 S_{2}(t)-S_{1}(t)\right] \\
S_{2}^{\prime}=\nu_{c}\left[3 S_{3}(t)-2 S_{2}(t)\right] \\
\cdots \\
S_{n}^{\prime}=\nu_{c}\left[(n+1) S_{n+1}(t)-n S_{n}(t)\right] \\
\cdots
\end{array}\right.
$$

Some explanations are in order at this level. Consider an $S$-individual with $n$ contacts. Let $\tau_{1}, \tau_{2}, \ldots, \tau_{n}$ be the random variable durations of contact. Then each random variable $\tau_{i}$ follows an exponential law with mean $1 / \nu_{c}$. Moreover, since $\tau_{1}, \tau_{2}, \ldots, \tau_{n}$ are independent random variables, the probability is

$$
\begin{gathered}
P\left(\tau_{1} \in[t,+\infty), \tau_{2} \in[t,+\infty), \ldots, \tau_{n} \in[t,+\infty)\right) \\
=\underbrace{\exp \left(-\nu_{c} t\right) \ldots \exp \left(-\nu_{c} t\right)}_{n \text { times }}=\exp \left(-n \nu_{c} t\right) .
\end{gathered}
$$

This justifies the term $-\nu_{V} n S_{n}(t)$ in the model, where $\nu_{V} n$ is the rate at which $S_{n}$-individuals are losing a contact with an $I$-individual.

\subsection{The SIR model}

The SIR model can be derived as before. Taking into account the fact that the flux of an $S$-individual losing one contact is given by $\nu_{c} \sum_{n=1}^{+\infty} n S_{n}(t)$, 
the flux of an $S$-individual becoming infective is given by $\nu_{c} p_{I} \sum_{n=1}^{+\infty} n S_{n}(t)$, and using Assumption 3.1 again, we obtain the following SIR model:

$$
\left\{\begin{aligned}
& S_{0}^{\prime}=\nu_{c}\left[\left(1-p_{I}\right) S_{1}(t)-\frac{I(t)}{N} S_{0}(t)\right]+\eta_{R} S_{1}(t) \\
& S_{1}^{\prime}=\nu_{c}\left[\frac{I(t)}{N} S_{0}(t)+\left(1-p_{I}\right) 2 S_{2}(t)-S_{1}(t)-\frac{I(t)}{N} S_{1}(t)\right]+\eta_{R}\left[2 S_{2}(t)-S_{1}(t)\right] \\
& \ldots \begin{array}{rl}
S_{n}^{\prime} & =\nu_{c}\left[\frac{I(t)}{N} S_{n-1}(t)+\left(1-p_{I}\right)(n+1) S_{n+1}(t)-n S_{n}(t)-\frac{I(t)}{N} S_{n}(t)\right] \\
& \quad+\eta_{R}\left[(n+1) S_{n+1}(t)-n S_{n}(t)\right]
\end{array} \\
& \quad \begin{array}{rl}
I^{\prime} & =\nu_{c} p_{I} \sum_{n=1}^{+\infty} n S_{n}(t)-\eta_{R} I(t)
\end{array} \\
& R^{\prime}=\eta_{R} I(t) .
\end{aligned}\right.
$$

The flowchart of model (4.4) is described in Fig. 10

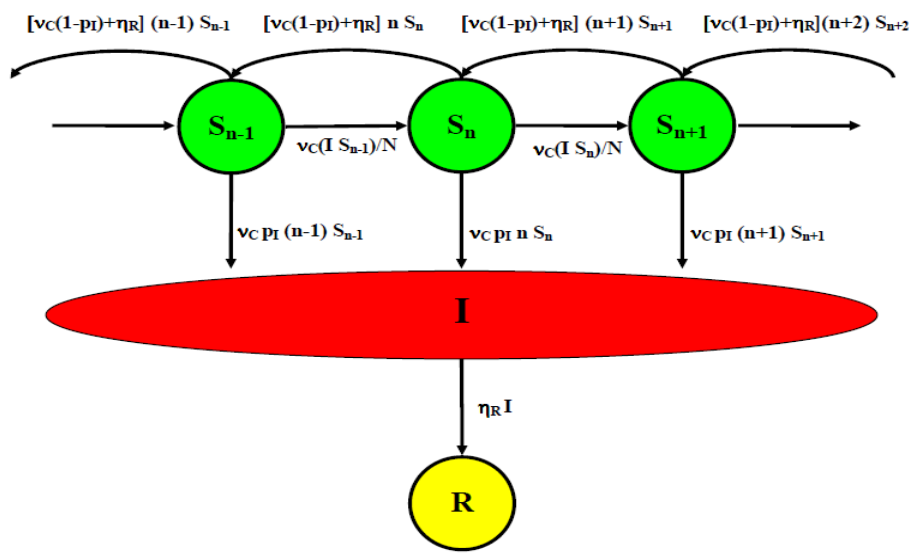

Figure 10: Flux diagram of model (4.4).

One may first observe that the SIR model (4.4) contains the usual term $\eta_{R} I$ in both the $I$-equation and the $R$-equation. This term describes the fact that the time spend by individuals in the infective class follows an exponential law with mean $T_{R}=1 / \eta_{R}$. However, since the $I$-individuals are becoming $R$-individuals, the numbers of $S_{n}$-individuals (for $n \geq 1$ ) are also affected by this process. Remembering that $S_{n}$ is the number of $S$-individuals who have been chosen $n$ times by an $I$-individual, the fact that some $I$-individuals are leaving will also induce a flux from the class $S_{n}$ into the class $S_{n-1}$. By using the same idea as the one used above for the loss of contacts, we deduce that the term $\eta_{R}\left[(n+1) S_{n+1}(t)-n S_{n}(t)\right]$ is needed in the $S_{n}$-equation. 
By renormalizing the distributions, namely by setting

$$
s_{n}=\frac{S_{n}}{N}, i=\frac{I}{N} \text { and } r=\frac{R}{N},
$$

we obtain the system

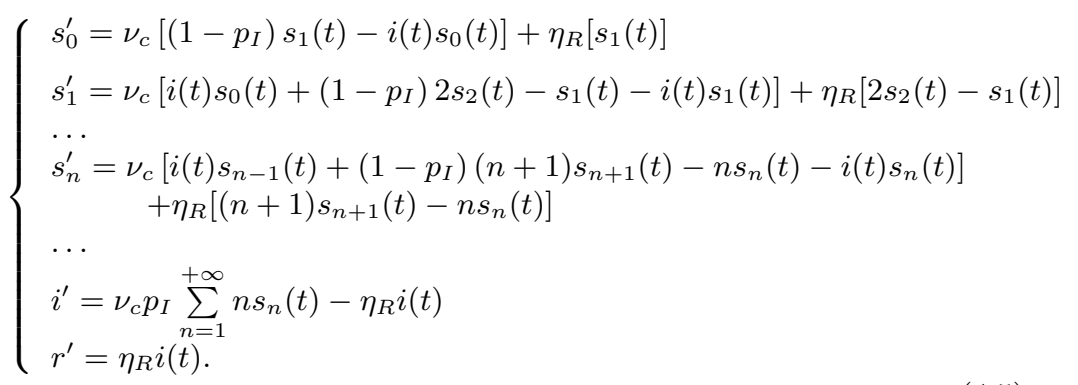

Moreover, we have

$$
s_{n} \geq 0, \forall n \geq 0,
$$

and

$$
\sum_{n=0}^{+\infty} s_{n}+i+r=1 .
$$

Furthermore, the quantity $\frac{\sum_{n=0}^{+\infty} n s_{n}}{\sum_{n=0}^{+\infty} s_{n}}$ is the average number with an $I$ individual. Therefore, it is natural to impose that

$$
\sum_{n=0}^{+\infty} n s_{n}<+\infty
$$

The analysis of model (4.5) will be presented elsewhere. We refer to Martcheva and Thieme [42] for results about the well posedness of similar classes of infinite differential equations.

\subsection{Formal singular limit to the classical SIR model}

By using again the rescalling

$$
\nu_{c}=\frac{1}{\varepsilon}, p_{I}=\beta_{I} \varepsilon,
$$

where $\varepsilon \in\left(0, \beta_{S}^{-1}\right]$ is supposed to be a small parameter of the system, then system (4.5) can be rewritten as

$$
\left\{\begin{array}{l}
s_{0}^{\prime}=\frac{1}{\varepsilon}\left[\left(1-\beta_{I} \varepsilon\right) s_{1}(t)-i(t) s_{0}(t)\right]+\eta_{R}\left[s_{1}(t)\right] \\
s_{1}^{\prime}=\frac{1}{\varepsilon}\left[i(t) s_{0}(t)+\left(1-\beta_{I} \varepsilon\right) 2 s_{2}(t)-s_{1}(t)-i(t) s_{1}(t)\right]+\eta_{R}\left[2 s_{2}(t)-s_{1}(t)\right] \\
\cdots \\
s_{n}^{\prime}=\frac{1}{\varepsilon}\left[i(t) s_{n-1}(t)+\left(1-\beta_{I} \varepsilon\right)(n+1) s_{n+1}(t)-n s_{n}(t)-i(t) s_{n}(t)\right] \\
\quad \quad+\eta_{R}\left[(n+1) s_{n+1}(t)-n s_{n}(t)\right] \\
\quad \ldots \quad \\
i^{\prime}=\beta_{I} \sum_{n=1}^{+\infty} n s_{n}(t)-\eta_{R} i(t) \\
r^{\prime}=\eta_{R} i(t) .
\end{array}\right.
$$


This singular perturbation problem describing the convergence of the above problem to the classical SIR model turns to be technical since

$$
\alpha_{\varepsilon}:=\left(1-\beta_{I} \varepsilon\right) \rightarrow 1 \text { as } \varepsilon(>0) \rightarrow 0 .
$$

Here we consider this problem formally.

Formal singular limit. Set $\varepsilon=0$ in system (4.6). Then we obtain

$$
\left\{\begin{array}{l}
0=\left[s_{1}(t)-i(t) s_{0}\right] \\
0=\left[i(t) s_{0}(t)+2 s_{2}(t)-s_{1}(t)-i(t) s_{1}(t)\right] \\
0=\left[i(t) s_{1}(t)+3 s_{3}(t)-2 s_{2}(t)-i(t) s_{2}(t)\right] \\
\cdots \\
0=\left[i(t) s_{n-1}(t)+(n+1) s_{n+1}(t)-n s_{n}(t)-i(t) s_{n}(t)\right] \\
\cdots \\
i^{\prime}=\beta_{I} \sum_{n=1}^{+\infty} n s_{n}(t)-\eta_{R} i(t) \\
r^{\prime}=\eta_{R} i(t) .
\end{array}\right.
$$

Hence the susceptible distribution must satisfy

$$
\left\{\begin{array}{l}
i s_{0}=s_{1} \\
(i+1) s_{1}=i s_{0}+2 s_{2} \\
(i+2) s_{2}=i s_{1}+3 s_{3} \\
\ldots \\
(i+n) s_{n}=i s_{n-1}+(n+1) s_{n+1} \\
\cdots
\end{array}\right.
$$

Therefore, we have

$$
\begin{aligned}
s_{1}= & i s_{0} \\
s_{2}= & \frac{1}{2}\left[(i+1) s_{1}-i s_{0}\right]=\frac{[(i+1) i-i]}{2} s_{0}=\frac{i^{2}}{2} s_{0} \\
s_{3}= & \frac{1}{3}\left[(i+2) s_{2}-i s_{1}\right]=\frac{1}{3}\left[(i+2) \frac{i^{2}}{2}-i^{2}\right]=\frac{i^{3}}{3 !} s_{0} \\
& \cdots \\
s_{n}= & \frac{1}{n}\left[(i+(n-1)) s_{n-1}-i s_{n-2}\right]=\frac{1}{n}\left[(i+(n-1)) \frac{i^{n-1}}{(n-1) !}-\frac{i^{n-1}}{(n-2) !}\right]=\frac{i^{n}}{n !} s_{0}
\end{aligned}
$$

By induction we obtain

$$
s_{n}=\frac{i^{n}}{n !} s_{0}, \forall n \geq 0 .
$$

Thus the fraction of susceptible is

$$
s:=\sum_{n=0}^{+\infty} s_{n}=\sum_{n=0}^{+\infty} \frac{i^{n}}{n !} s_{0}=s_{0} \exp (i) .
$$

Since $s+i+r=1$, we obtain

$$
s_{0}=s \exp (-i)=(1-i-r) \exp (-i) .
$$


It follows that $\frac{s_{n}}{s}$ is a Poisson distribution with parameter $i$, which means that

$$
\frac{s_{n}}{s}=\frac{i^{n}}{n !} \exp (-i), \forall n \geq 0 .
$$

We can interpret the quantity

$$
s_{n}=\frac{i^{n}}{n !}(1-(i+r)) \exp (-i)
$$

as the probability that an $S$-individual has been chosen by $n I$-individuals.

Since $s_{n}$ is the proportion of $S$-individuals which have been chosen by $n I$-individuals, the average number of contacts per $S$-individual is

$$
\sum_{n=1}^{+\infty} n s_{n}=\left(\sum_{n=0}^{+\infty} \frac{i^{n}}{n !} s_{0}\right) i=s i
$$

Hence, formally as the singular limit, we obtain the SIR type model

$$
\left\{\begin{array}{l}
s^{\prime}=-\beta_{I} s i \\
i^{\prime}=\beta_{I} s i-\eta_{R} i \\
r^{\prime}=\eta_{R} i
\end{array}\right.
$$

\subsection{Numerical simulations}

In order to perform numerical simulations of system (4.4), we truncate the system at the order $\left(n^{*}+1\right)>1$. That is, we neglect the terms $S_{k}^{\prime}(t)$ for $k \geq\left(n^{*}+2\right)$ and consider the following system:

$$
\left\{\begin{array}{l}
S_{0}^{\prime}=\nu_{c}\left[\left(1-p_{I}\right) S_{1}(t)-\frac{I(t)}{N} S_{0}(t)\right]+\eta_{R} S_{1}(t) \\
S_{1}^{\prime}=\nu_{c}\left[\frac{I(t)}{N} S_{0}(t)+\left(1-p_{I}\right) 2 S_{2}(t)-S_{1}(t)-\frac{I(t)}{N} S_{1}(t)\right]+\eta_{R}\left[2 S_{2}(t)-S_{1}(t)\right] \\
\cdots \\
S_{n^{*}-1}^{\prime}=\nu_{c}\left[\frac{I(t)}{N} S_{n^{*}-2}(t)+\left(1-p_{I}\right) n^{*} S_{n^{*}}(t)-\left(n^{*}-1\right) S_{n^{*}-1}(t)-\frac{I(t)}{N} S_{n^{*}-1}(t)\right] \\
\quad+\eta_{R}\left[n^{*} S_{n^{*}}(t)-\left(n^{*}-1\right) S_{n^{*}-1}(t)\right] \\
S_{n^{*}}^{\prime}=\nu_{c}\left[\frac{I(t)}{N} S_{n^{*}-1}(t)-n^{*} S_{n^{*}}(t)-\frac{I(t)}{N} S_{n^{*}}(t)\right]-\eta_{R}\left[n^{*} S_{n^{*}}(t)\right] \\
I^{\prime}=\nu_{c} p_{I} \sum_{k=1}^{n^{*}} k S_{k}(t)-\eta_{R} I(t) \\
R^{\prime}=\eta_{R} I(t) .
\end{array}\right.
$$

At time $t=0$, we will take the following initial distributions to simulate the above model

$$
N>I>0, R=0,
$$

and

$$
S_{n}=N \frac{i^{n}}{n !} \exp (-i)(1-i), \forall n=0, \ldots, n^{*},
$$

with

$$
i=\frac{I}{N} \text {. }
$$

As before, we need to specify further the rules of transmission between an $S$-individual and an $I$-individual. 
Assumption 4.1 We further assume that

(a) (New SIR model) For a given pair of $(S, I)$-individuals in contact, if the individual initiating the contact is an $I$-individual, then the transmission only occurs at the end of the contact with the probability $p_{I}$.

(b) (Classical SIR Model) For a given pair of $(S, I)$-individuals in contact, if the individual initiating the contact is an $I$-individual, then the transmission only occurs at the beginning of the contact with the probability $p_{I}$.

We will use Assumption 4.1(a) to run an IBM (called here IBM $_{12}$ ) which corresponds to the new model (4.10), while Assumption 4.1(b) will be used to run an IBM (called here $\mathrm{IBM}_{22}$ ) which corresponds to the classical SIR model (1.1). The comparison between the new model (4.10) and the classical SIR model (1.1) with $\beta=\beta_{I}$ is given in Fig. 10. We can observe numerically the convergence of the new SIR model (4.10) to the classical SIR model (1.1) when $T_{C}$ goes to zero and $p_{I}=\beta_{I} T_{C}$. Similarly, we can also see that the curve of the recovereds is influenced by the parameter $T_{C}$. Let $R_{\infty}$ the limit of $R(t)$ when $t$ goes $+\infty$. In (a) the total numbers of cases for the new SIR model (4.10) and the classical SIR model (1.1) are different. Once again one must be very careful in using IBM to simulate an epidemic, since replacing one model by the other might lead to a large bias in prediction. Here $S / N=0.9, I / N=0.1$, $S_{c}=I_{c}=S I / N$ at time $t=0 . T_{R}=10$ and $\beta_{I}=0.2$ are fixed and in (a) (resp. (b)) $T_{C}=5$ and $p_{I}=\beta_{I} T_{C}=1$ (resp. $T_{C}=0.5$ and $\left.p_{I}=\beta_{I} T_{C}=0.1\right)$ are also fixed.

(a)

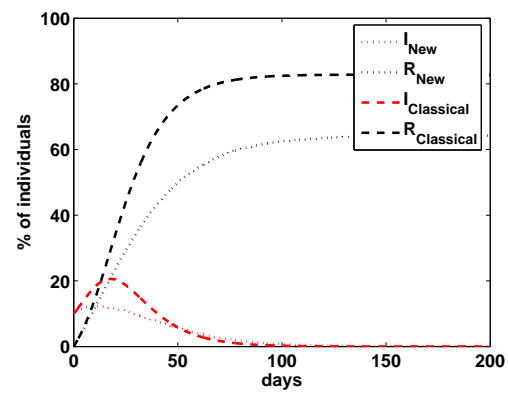

(b)

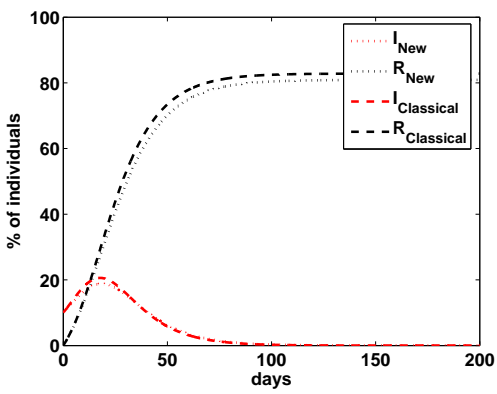

Figure 11: Comparison of the new SIR model (4.10) (dotted curves) and the classical SIR model (1.1) (dashed curves) with $\beta=\beta_{S}$. (a) The total number of recovered individuals in the new and classical SIR models are significantly different as time increases. (b) The solution of the new SIR model converges to the solution of the classical SIR model. Here $S / N=0.9, I / N=0.1, S_{c}=I_{c}=$ $S I / N$ at time $t=0, T_{R}=10$ and $\beta_{I}=0.2$ (a) $T_{C}=5$ and $p_{I}=\beta_{I} T_{C}=1$. (b) $T_{C}=0.5$ and $p_{I}=\beta_{I} T_{C}=0.1$

The comparison of the IBM, the new SIR model (4.10), and the classical SIR model (1.1) is given in Fig. 12. Here $S / N=0.9, I / N=0.1$, 
$S_{c}=I_{c}=S I / N$ at time $t=0 . T_{R}=10, \beta_{I}=0.2, T_{C}=5$, and $p_{I}=\beta_{I} T_{C}=1$ are fixed. In (a) (resp. (b)) the $\mathrm{IBM}_{12}$ was run to simulate the transmission at the end of the contact for $N=100$ (resp. $N=10000$ ). In (c) (resp. (d)) the $\mathrm{IBM}_{22}$ was run to simulate the transmission at the beginning of the contact for $N=100$ (resp. $N=10000$ ).

(a)

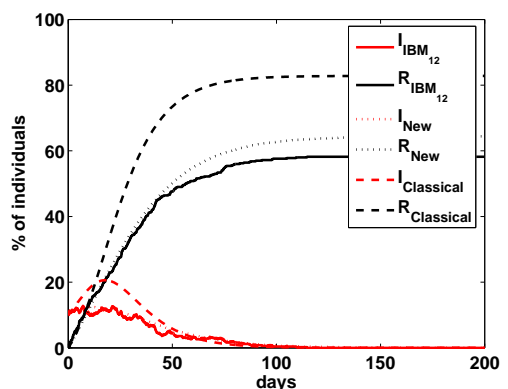

(c)

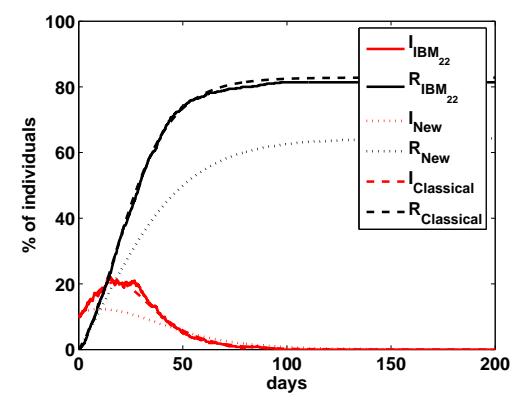

(b)

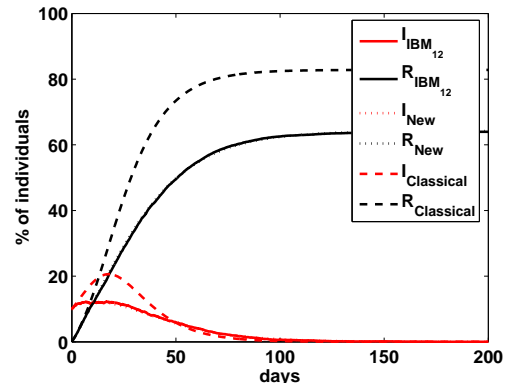

(d)

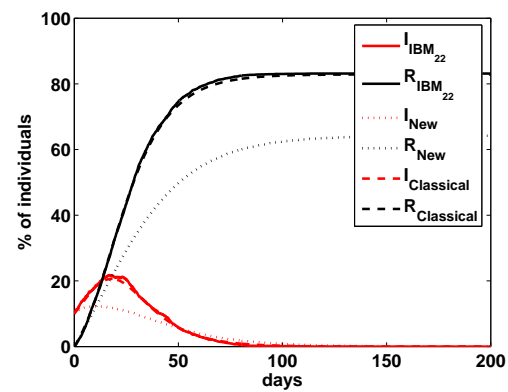

Figure 12: Comparison of the IBM (solid curves), the new SIR model (4.10) (dotted curves), and the classical SIR model (1.1) (dashed curves) with $\beta=$ $\beta_{I}, S / N=0.9, I / N=0.1, S_{c}=I_{c}=S I / N$ at time $t=0 . T_{R}=10, \beta_{I}=0.2$, $T_{C}=5$, and $p_{I}=\beta_{I} T_{C}=1$ are fixed. In (a) and (b) the $\mathrm{IBM}_{12}$ was run to simulate the transmission at the end of the contact for $N=100$ and $N=10000$, respectively. In (c) and (d) the $\mathrm{IBM}_{22}$ was run to simulate the transmission at the beginning of the contact for $N=100$ and $N=10000$, respectively.

\section{Discussion}

Stochastic individual-based models (IBM) use continuum dynamics to track relatively small numbers of individuals based on the change rates of time and view individuals as individual agents whose status changes based on probabilistic events occurring over time. Such models are particularly suitable to describe the transmission dynamics of infectious diseases in a small population in which the individual behavior plays an important role in the spread of diseases. Most stochastic IBM simulations are based on the framework of certain deterministic epidemic models, in particular the 
classical susceptible-infective-recovered (SIR) model.

The purpose of this article was to compare the stochastic IBM and the classical SIR model and to examine how the behavior at the individual level affects the eventual transmission dynamics of infectious diseases at the population level. We first made some assumptions about the rules of contacts between susceptible and infective individuals, the rules of transmission of diseases through these contacts, and the time of transmission during contacts. For the sake of comparison, we distinguished two types of transmission processes: that initiated by susceptible individuals and that driven by infective individuals. We then studied the transmission driven only by the susceptible individuals and compared the numerical simulations between the IBM and the classical SIR models. The transmission initiated only by infective individuals was also modeled and analyzed and the comparison of stochastic IBM simulations and the classical SIR model was presented. Our analysis and simulations demonstrate that the IBM converges to the classical SIR model only in some particular situations. In general, the individual-based and the classical SIR models are significantly different. Moreover, our study reveals that the timing of transmission in a contact at the individual level plays a crucial role in the transmission dynamics of a disease at the population level.

Stochastic SIR epidemics models have been studied extensively and we refer to Allen [2, 3], Anderson and Britton [5], and Britton [12] (and references therein) for details on stochastic epidemic models. However, as far as we understand the derivations of most of these stochastic SIR models are not very clear and poorly understood using individual stochastic rules. This is left for future consideration.

There are several issues which deserve further investigation. Firstly, the stochastic process introduced in section 2 needs to be studied in detail. Secondly, we assumed that the time of transmission of diseases was either at the beginning or at the end of a contact, in reality the time of transmission probably occurs at certain moment between the beginning and the end of a contact. Therefore, modeling the disease transmission with such a timing of transmission and comparing these models is useful. Thirdly, both the spatial structure and the age of infection play very important roles in the spread of infectious diseases, it will be very interesting to include these features into the individual stochastic modeling. Finally, since the contact network is dynamical and fully random, the results will change if other graphs of contact are used for the stochastic simulations. We plan to consider some individual-based models for other types of contact networks in future studies.

Acknowledgments. We would like to thank Professor Donald DeAngelis and Professor Jacques Demongeot for reading the manuscript and making comments and corrections. We also thank the referees for their helpful comments and suggestions. 


\section{References}

[1] M. Ajelli, B. Gonalves, D. Balcan, V. Colizza, H. Hu, J. J. Ramasco, S. Merler and A. Vespignani, RCesoeamrchp artaicrleing large-scale computational approaches to epidemic modeling: Agent-based versus structured metapopulation models, BMC Inf. Dis. 10 (2010): 190. http://www.biomedcentral.com/1471-2334/10/190.

[2] L. J. S. Allen, An Introduction to Stochastic Processes with Applications to Biology, Prentice Hall, NJ, 2003.

[3] L. J. S. Allen, An Introduction to Stochastic Epidemic Models, in "Mathematical Epidemiology", Lecture Notes in Math. 1945, F. Brauer, P. van den Driessche, and J. Wu (eds.), Springer, New York, 2008, pp. 81-130.

[4] R. M. Anderson and R. M. May, Infective Diseases of Humans: Dynamics and Control, Oxford University Press, Oxford, 1991.

[5] H. Andersson and T. Britton, Stochastic Epidemic Models and Their Statistical Analysis. Lecture Notes in Stat. 151, Springer-Verlag, New York, 2000.

[6] J. Arino, Diseases in metapopulations, in "Modeling and Dynamics of Infectious Diseases", Z. Ma, Y. Zhou, and J. Wu (eds.), World Scientific, Singapore, 2009.

[7] N. T. J. Bailey, The Mathematical Theory of Epidemics, Charles Griffin, London, 1957.

[8] A. Barrat, M. Bathélemy and A. Vespignani, Dynamical Processes on Complex Networks, Cambridge University Press, Cambridge, 2008.

[9] M. Bartlett, Stochastic Population Models in Ecology and Epidemiology, Methuen, London, 1960.

[10] D. Bernoulli, Essai d'une nouvelle analyse de la mortalité causée par la petite vérole et des avantages de l'inoculation pour la prévenir, Mém. Math. Phys. Acad. Roy. Sci., Paris (1760), 1-45.

[11] F. Brauer and C. Castillo-Chavez, Mathematical Models in Population Biology and Epidemiology, Springer, New York, 2000.

[12] T. Britton, Stochastic epidemic models: a survey, Math. Biosc. 225 (2010), 24-35.

[13] S. Busenberg and K. Cooke, Vertically Transmitted Diseases: Models and Dynamics. Lecture Notes in Biomath. 23, Springer-Verlag, Berlin, 1993.

[14] V. Capasso, Mathematical Structures of Epidemic Systems, Lecture Notes in Biomath. 97, Springer-Verlag, Heidelberg, 1993.

[15] Centers for Disease Control and Prevention, Measles outbreakNetherlands, April 1999-January 2000, MMWR, 49 (2000), 299-303.

[16] D. J. Daley and J. Gani, Epidemic Modelling An Introduction, Cambridge Studies Math. Biol. 15, Cambridge University Press, Cambridge, 1999 . 
[17] E. M. C. D'Agata, P. Magal, D. Olivier, S. Ruan, G. F. Webb, Modeling antibiotic resistance in hospitals: The impact of minimizing treatment duration, J. Theoret. Biol. 249 (2007), 487-499.

[18] D. L. DeAngelis and W. M. Mooij, Individual-based modeling of ecological and evolutionary processes, Annu. Rev. Ecol. Evol. Syst. 36 (2005), 147-168.

[19] O. Diekmann and J. A. P. Heesterbeek Mathematical Epidemiology of Infectious Diseases: Model Building, Analysis and Interpretation, Wiley, Chichester, 2000.

[20] K. Dietz and J. A. P. Heesterbeek, Bernoulli was ahead of modern epidemiology, Nature 408 (2000), 513-514.

[21] K. Dietz and J. A. P. Heesterbeek, Daniel Bernoulli's epidemiological model revisited, Math. Biosci. 180 (2002), 1-21.

[22] J. L. Doob, Topics in the theory of Markoff chains, Trans. Amer. Math. Soc. 52 (1942), 37-64.

[23] J. L. Doob, Markoff chains - Denumerable case, Trans. Amer. Math. Soc. 58 (1945), 455-473.

[24] R. Durrett, Random Graph Dynamics, Cambridge University Press, Cambridge, 2007.

[25] R. Durrett, Some features of the spread of epidemics and information on a random graph, Proc. Natl Acad. Sci. USA 107 (2010), 44914498.

[26] R. Durrett and S. A. Levin, The importance of being discrete (and spatial), Theoret. Pop. Biol. 46 (1994), 363-394.

[27] D. T. Gillespie, A general method for numerically simulating the stochastic time evolution of coupled chemical reactions, J. Computat. Phys. 22 (1976), 403-434.

[28] D. T. Gillespie, Exact stochastic simulation of coupled chemical reactions, J. Phys.Chem. 81 (1977), 2340-2361.

[29] V. Grimm and S. F. Railsback, Individual-based Modeling and Ecology, Princeton University Press, Princeton, 2005.

[30] H. W. Hethcote, The mathematics of infectious diseases, SIAM Rev. 42 (2000), 599-653.

[31] J. A. Higgins, S. Hoffman and S. L. Dworkin, Rethinking gender, heterosexual men, and women's vulnerability to HIV/AIDS, Amer. J. Public Health 100 (2010), 435-445.

[32] P. Hinow, F. Le Foll, P. Magal, G. F. Webb, Analysis of a model for transfer phenomena in biological populations, SIAM J. Appl. Math. 70 (2009), 40-62.

[33] M. Iannelli, Mathematical Theory of Age-structured Population Dynamics, Applied Mathematics Monographs CNR 7, Giadini Editori e Stampatori, Pisa, 1994.

[34] M. J. Keeling and B. T. Grenfell, Individual-based perspectives on $R_{0}, J$. Theor. Biol. 203 (2000), 51-61. 
[35] M. J. Keeling and P. Rohani, Modeling Infectious Diseases in Humans and Animals, Princeton University Press, Princeton, 2007.

[36] W. O. Kermack and A. G. McKendrick, A contribution to the mathematical theory of epidemics, Proc. R. Soc. Lond. A 115 (1927), 700-721.

[37] W. O. Kermack and A. G. McKendrick, Contributions to the mathematical theory of epidemics: II, Proc. R. Soc. Lond. A 138 (1932), $55-83$.

[38] W. O. Kermack and A. G. McKendrick, Contributions to the mathematical theory of epidemics: III, Proc. R. Soc. Lond. A 141 (1933), 94-112.

[39] T. G. Kurtz, Approximation of Population Processes, Society for Industrial and Applied Mathematics, Philadelphia, 1981.

[40] S. A. Levin and R. Durrett, From individuals to epidemics, Phil. Trans R. Soc. Lond. B 351 (1996), 1615-1621.

[41] P. Magal, C. C. McCluskey, and G. F. Webb, Liapunov functional and global asymptotic stability for an infection-age model, Appl. Anal. 89 (2010), 1109-1140.

[42] M. Martcheva and H. R. Thieme, Infinite ODE systems modeling size-structured metapopulations, macroparasitic diseases, and prion proliferation, in "Structured Population Models in Biology and Epidemiology", P. Magal and S. Ruan (eds.), Lecture Notes in Math. 1936, Springer, Berlin, 2008, 51-113.

[43] L. A. Meyers, Contact network epidemiology: Bond percolation applied to infectious disease prediction and control, Bull. Amer. Math. Soc. 44 (2007), 63-86.

[44] L. A. Meyers, M. E. J. Newman, and B. Pourbohloul, Predicting epidemics on directed contact networks, Journal of Theoretical Biology 240 (2006), 400-418.

[45] H. Muench, Catalytic Models in Epidemiology, Harvard University Press, Cambridge, 1959.

[46] J. D. Murray, Mathematical Biology, Springer, Berlin, 1993.

[47] C. J. Mode and C. K. Sleeman, Stochastic Processes in Epidemiology. HIV/AIDS, Other Infectious Diseases and Computers, World Scientific, Singapore, 2000.

[48] M. E. J. Newman, The structure and function of complex networks, SIAM Rev. 45 (2003), 167-256.

[49] M. Pascual and S. A. Levin, From individuals to population densities: Searching for the intermediate scale of nontrivial determinism, Ecology 80 (1999), 2225-2236.

[50] L. Rass and J. Radcliffe, Spatial Deterministic Epidemics, Math. Surveys Monogr. 102, Amer. Math. Soc., Providence, RI, 2003.

[51] R. Ross, An application of the theory of probabilities to the study of a priori pathometry: I, Proc. R. Soc. Lond. A 92 (1916), 204-230. 
[52] R. Ross and H. P. Hudson, An application of the theory of probabilities to the study of a priori pathometry: II, Proc. R. Soc. Lond. A 93 (1917), 212-225.

[53] R. Ross and H. P. Hudson, An application of the theory of probabilities to the study of a priori pathometry: III, Proc. R. Soc. Lond. A 93 (1917), 225-240.

[54] S. Ruan, Spatial-Temporal Dynamics in Nonlocal Epidemiological Models, in "Mathematics for Life Science and Medicine", Y. Takeuchi, K. Sato and Y. Iwasa (eds.), Springer-Verlag, Berlin, 2007, pp. 99-122.

[55] S. Ruan and J. Wu, Modeling Spatial Spread of Communicable Diseases Involving Animal Hosts, in "Spatial Ecology", S. Cantrell, C. Cosner and S. Ruan (eds.), Chapman \& Hall/CRC, Boca Raton, FL, 2009, pp. 293-316.

[56] K. J. Sharkey, Deterministic epidemiological models at the individual level, J. Math. Biol. 57 (2008), 311-331.

[57] T. Smieszek, L. Fiebig and R. W. Scholz, Models of epidemics: When contact repetition and clustering should be included, Theoret. Biol. Med. Model. 6 (2009): 11. doi:10.1186/1742-4682-6-11.

[58] H. L. Smith, Monotone Dynamical Systems: An Introduction to the Theory of Competitive and Cooperative Systems, Math. Surveys Monogr. 41, Amer. Math. Soc., Providence, RI, 1995.

[59] H. R. Thieme, Mathematics in Population Biology, Princeton University Press, Princeton, 2003.

[60] H. R. Thieme and C. Castillo-Chavez, How may infection-agedependent infectivity affect the dynamics of HIV/AIDS? SIAM. J. Appl. Math. 53 (1993), 1447-1479.

[61] G. F. Webb, Theory of Nonlinear Age-dependent Population Dynamics, Marcel Dekker, New York, 1985. 